\title{
General Method for Calculating the Response and Noise Spectra of Active Fabry-Perot Semiconductor Waveguides With External Optical Injection
}

Blaaberg, Søren; Mørk, Jesper

Published in:

I E E E Journal of Quantum Electronics

Link to article, DOI:

10.1109/JQE.2009.2014690

Publication date:

2009

Document Version

Publisher's PDF, also known as Version of record

Link back to DTU Orbit

Citation (APA):

Blaaberg, S., \& Mørk, J. (2009). General Method for Calculating the Response and Noise Spectra of Active Fabry-Perot Semiconductor Waveguides With External Optical Injection. I E E E Journal of Quantum Electronics, 45(8), 940-953. https://doi.org/10.1109/JQE.2009.2014690

\section{General rights}

Copyright and moral rights for the publications made accessible in the public portal are retained by the authors and/or other copyright owners and it is a condition of accessing publications that users recognise and abide by the legal requirements associated with these rights.

- Users may download and print one copy of any publication from the public portal for the purpose of private study or research.

- You may not further distribute the material or use it for any profit-making activity or commercial gain

- You may freely distribute the URL identifying the publication in the public portal 


\title{
General Method for Calculating the Response and Noise Spectra of Active Fabry-Perot Semiconductor Waveguides With External Optical Injection
}

\author{
Søren Blaaberg and Jesper Mørk
}

\begin{abstract}
We present a theoretical method for calculating small-signal modulation responses and noise spectra of active Fabry-Perot semiconductor waveguides with external light injection. Small-signal responses due to either a modulation of the pump current or due to an optical amplitude or phase modulation of the input field can be calculated. Both responses and noise spectra are given through semianalytical expressions taking into account the longitudinal extent and finite end-facet reflectivities of the active device. Different examples of responses and spectra are presented for semiconductor optical amplifiers and an injection-locked laser. We also demonstrate the applicability of the method to analyze slow and fast light effects in semiconductor waveguides. Finite reflectivities of the facets are found to influence the phase changes of the injected microwave-modulated light.
\end{abstract}

Index Terms-Fast light, Green function, injection locking, semiconductor lasers, semiconductor optical amplifiers (SOAs), slow light.

\section{INTRODUCTION}

M ODULATION responses of optical semiconductor devices subject to injected light are of interest in, e.g., microwave photonics and in optical communications. Direct modulation of the current of injection-locked semiconductor lasers may be used for generation of pulse trains or for direct data encoding in optical communications. Direct phase modulation [1] and amplitude modulation with a suppressed magnitude of chirp [2] are well-known properties for injection-locked lasers. In addition, the modulation bandwidth in injection-locked semiconductor lasers can be enhanced compared with their solitary counterparts [3], and the frequency of the response resonance may be shifted [3], [4] due to the injection of light. Also, direct current modulation of SOAs has been investigated [5], [6]. Recently, there has been a growing interest in responses due to optical modulation of a signal injected into semiconductor devices. Slow and fast light effects in active semiconductor waveguides [7]-[9] can be utilized to impose phase changes on an injected RF-intensity modulated optical signal, suitable for applications in microwave photonics. Slow and fast light are caused by wave mixing in the semiconductor when a single RF-intensity modulated optical carrier signal is injected into an active waveguide.

Manuscript received August 29, 2008; revised January 02, 2009. Current version published July 01, 2009.

The authors are with DTU Fotonik, Department of Photonics Engineering, Technical University of Denmark, Kgs. Lyngby, DK-2800, Denmark (e-mail: sblj@fotonik.dtu.dk).

Digital Object Identifier 10.1109/JQE.2009.2014690
One can interpret slow and fast light as a phase shift of the RF envelope with a magnitude controlled by, e.g., the applied dc pump current or bias voltage.

Fluctuations due to spontaneous emission noise and charge carrier noise are potential sources for disturbances of the injected signal; see [10] for a recent detailed treatment. The relative intensity noise (RIN) spectrum can, e.g., be of importance in applications such as phase-arrayed devices. Shtaif et al. [11] calculated noise spectra for an SOA assuming perfectly anti-reflection (AR)-coated end facets.

Recently, there has been a renewed interest in the modulation response of injection-locked lasers. As already mentioned, the bandwidth properties of a semiconductor laser can be modified when injection-locked as a slave laser. When the injected field from the master laser has a frequency detuned from the cavity mode(s) of the slave laser, enhanced modulation response may be seen at modulation frequencies equal to the detuning of the master laser from the shifted cavity modes in the optical spectrum. Usually, when the slave laser is relatively short, only one cavity mode is relevant as the longitudinal mode spacing is too large for secondary modes to play a role. However, for relatively long slave lasers, more than one mode can become important. Commonly, theoretical work on injection-locked semiconductor lasers has been done using a lumped description of the slave laser [12]-[14] not resolving the laser axis and thus only including one cavity mode. Modulation responses both due to current modulation [15] and RF-intensity modulation of the injected optical signal [16] are of current interest. In [16], RF-phase shifts of intensity modulated signals in injectionlocked slave VCSELS were investigated and large RF-phase shifts of 360 degrees were found, albeit not continuously tunable from 0 to 360 degrees.

The intention of this paper is to present a general method to obtain small-signal responses and noise spectra in active semiconductor devices with finite facet reflectivities and subject to injected light. The method allows for theoretical studies of devices where reflective end facets are necessary, e.g., injectionlocked lasers and reflective SOAs [17], as well as devices where poorly AR-coated facets, may alter the performance of the device in an undesired way. A major part of the presented numerical examples concerning modulation responses address cases where the input field is optically modulated while holding the pump current constant. In coherent communications and RF photonics, the conversion from one optical modulation format to another, for example, phase to amplitude, is of interest [18]. As we discuss in this paper, a phase-modulated input signal will 


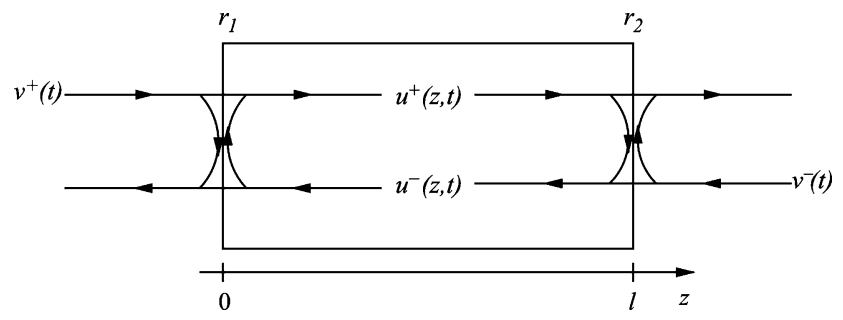

Fig. 1. Diagram of a Fabry-Perot amplifier or laser with external incident fields $v^{ \pm}$and intra-cavity fields $u^{ \pm}$. Amplitude reflectivities of end facets are denoted $r_{1}$ and $r_{2}$.

only give rise to a device response if reflections occur at the facets.

This paper is organized as follows. In Section II, the basic equations for the field and carrier density are given. Then, in Section III, the mathematical formalism of the small-signal analysis are discussed. The small-signal analysis relies on the Green's function method presented for DFB lasers in [19]. Due to injection of light into the active semiconductor waveguide the boundary conditions differ from the case of a solitary laser, implying modifications of the semianalytical solutions to the linearized field equations. In Section IV, expressions for modulation responses are given explicitly in terms of functions found in the previous section. Examples of calculated modulation responses for an SOA are presented in Section V. Noise spectra are formulated in Section VI and examples for SOAs given in Section VII. With sufficiently high end-facet reflectivities the active waveguide may act as an injection-locked laser for which examples of responses and spectra are given in Section VIII. Finally, Section IX presents the main conclusions.

\section{BASIC EQUATIONS}

We consider the generic structure in Fig. 1 consisting of a onedimensional (1-D) active semiconductor waveguide of length $l$ with end facets at $z=0$ and $z=l$ with amplitude reflectivities $r_{1}$ and $r_{2}$, respectively. The complex electric field $E(t, z)$ inside the waveguide is assumed to be a sum of a forward and a backward traveling wave, i.e., $E(t, z)=E^{+}(t, z)+E^{-}(t, z)$. Using envelope functions $u^{ \pm}(t, z)$, the fields are written as $E^{ \pm}(t, z)=$ $u^{ \pm}(t, z) \exp \left[j\left(\omega_{s} t \mp k_{r} z\right)\right]$ with the carrier frequency of the incident signal $\omega_{s}$ and a reference wavenumber $k_{r}=\omega_{r} n_{r} / c=$ $2 \pi n_{r} / \lambda_{r}$, where $\omega_{r}\left(\lambda_{r}\right)$ is a reference frequency (wavelength), $n_{r}$ is the modal refractive index at $\omega_{r}$, and $c$ is the vacuum speed of light. Incident on the end facets are the external fields $v^{+}$and $v^{-}$.

In the time domain, the field equations for the signal inside the device are given as [19]

$\frac{\partial}{\partial z} u^{ \pm}(t, z) \pm \frac{1}{v_{g}} \frac{\partial}{\partial t} u^{ \pm}(t, z) \pm j\left(k-k_{r}\right) u^{ \pm}(t, z)=f^{ \pm}(t, z)$ where $k(\omega, N, S)$ is the complex wavenumber, which is in general a function of optical frequency $\omega$, carrier density $N$, and photon density $S$, here taken at the optical frequency $\omega_{s}$ as

$$
k=k_{r}+\frac{1}{v_{g}} 2 \pi \Delta+\frac{1}{2}(j-\alpha) g\left(\omega_{s}, N, S\right)-\frac{j}{2} \alpha_{i}
$$

Here, $v_{g}$ is the group velocity, $\alpha$ is the linewidth enhancement factor, $g$ is the modal gain, $\alpha_{i}$ is the internal loss, and $\Delta=$ $\left(\omega_{s}-\omega_{r}\right) / 2 \pi$ is the detuning. The Langevin noise terms $f^{ \pm}$ are due to spontaneous emission noise. In (2), it has been assumed that the signal is sufficiently narrowband for gain dispersion to be neglected. The particular gain model used for the example calculations in this paper is described in Appendix A. The boundary conditions for $u^{ \pm}$at the end facets are given as

$$
\begin{aligned}
u^{+}(0, t) & =r_{1} u^{-}(0, t)+t_{l r} v^{+}(t) \\
u^{-}(l, t) e^{j k_{r} l} & =r_{2} e^{-j k_{r} l} u^{+}(l, t)+t_{r l} v^{-}(t) .
\end{aligned}
$$

where $v^{ \pm}$are external fields incident on the left and right facets, $r_{1}\left(r_{2}\right)$ is the reflection coefficient of the left (right) facet, and $t_{l r}\left(t_{l r}\right)$ is the transmission coefficient of the input field incident on the left (right) facet. Energy conservation implies $\left|t_{l r}\right|^{2}=$ $\left(1-\left|r_{1}\right|^{2}\right) / n_{r}$ and $\left|t_{r l}\right|^{2}=\left(1-\left|r_{2}\right|^{2}\right) / n_{r}$. The relation between photon densities $S^{ \pm}$and field envelopes $u^{ \pm}$is

$$
S^{ \pm}(t, z)=\frac{2 \epsilon_{0} n_{r} n_{g}}{\hbar \omega A_{\mathrm{ph}}}\left|u^{ \pm}(t, z)\right|^{2} .
$$

Neglecting beating and standing wave effects the total signal photon density $S$ becomes $S(t, z)=S^{+}(t, z)+S^{-}(t, z) . A_{c}$ is the transverse area of the active layer and the area $A_{\mathrm{ph}}=A_{c} / \Gamma$ is the cross-sectional area of the transverse photon distribution with the confinement factor $\Gamma . n_{g}$ is the effective group index. The fields $u^{ \pm}(t, z)$ have unit $\mathrm{V}$ and the photon densities have unit $\mathrm{m}^{-3}$.

We consider the general case where the carrier density varies along the $z$-axis, as it is the case when the amplifier is neither unsaturated nor completely saturated. The rate equation for the carrier density can be stated as

$$
\frac{\partial}{\partial t} N=J-R_{\mathrm{sp}}-R_{\mathrm{st}}-R_{\mathrm{ASE}}+F_{N}
$$

where $J$ is the pump rate, $R_{\mathrm{sp}}(N)$ is the recombination rate due to spontaneous emmision and nonradiative recombination, while $R_{\mathrm{st}}$ is the stimulated emission rate due to signal photons and $F_{N}$ is the Langevin driving term representing the carrier noise. The recombination rates used here are

$$
\begin{aligned}
R_{\mathrm{sp}} & =A N+B N^{2}+C N^{3} \\
R_{\mathrm{st}} & =v_{g} \frac{g\left(N, \omega_{s}, S\right)}{\Gamma} S .
\end{aligned}
$$

The term $R_{\mathrm{ASE}}$ is the stimulated emission rate due to amplified spontaneous emission (ASE) causing gain saturation and is described in Appendix A. $R_{\mathrm{ASE}}$ is significant only at low input 
powers. The signal output powers $P_{L}$ and $P_{R}$ from the left and right facets are written as

$$
\begin{aligned}
& P_{L}(t)=2 \epsilon_{0} n_{r} c\left(1-r_{1}^{2}\right)\left|u^{-}(0, t)\right|^{2}+r_{1}^{2} P_{\text {in }}^{+}(t) \\
& P_{R}(t)=2 \epsilon_{0} n_{r} c\left(1-r_{2}^{2}\right)\left|u^{+}(l, t)\right|^{2}+r_{2}^{2} P_{\text {in }}^{-}(t) .
\end{aligned}
$$

Here, the effective index experienced by the signal has been approximated to be $n_{r}$ while the relation between the input fields $v^{ \pm}$and the left and right input powers just outside the end facets are

$$
\begin{aligned}
& P_{\text {in }}^{+}(t)=2 \epsilon_{0} c\left|v^{+}(t)\right|^{2} \\
& P_{\text {in }}^{-}(t)=2 \epsilon_{0} c\left|v^{-}(t)\right|^{2} .
\end{aligned}
$$

For $f=F_{N}=0$ and a steady pump distribution $J(t, z)=J_{s}(z)$, we can calculate stationary solutions $\left\{u_{s}^{ \pm}(z), N_{s}(z)\right\}$ from the expressions (1) and (5) for given stationary input fields $v_{s}^{ \pm}$using standard methods [20]. A small-signal analysis may then be performed around stable stationary solutions as described in subsequent sections.

\section{SMALl-Signal ANALYSIS}

A linear expansion of the field equation (1) and the carrier equation (5) around a stationary solution consisting of a stationary field distribution $u_{s}^{ \pm}(z)$ and a stationary carrier distribution $N_{s}(z)$ forms the basis of the small-signal analysis [19]. Thus, $u^{ \pm}(t, z) \simeq u_{s}^{ \pm}(z)+\delta u^{ \pm}(t, z)$ and $N(t, z) \simeq N_{s}(z)+$ $\delta N(t, z)$. With real amplitudes $a^{ \pm}(t, z)$ and phases $\varphi^{ \pm}(t, z)$, the field envelopes are written as $u^{ \pm}(t, z)=a^{ \pm} \exp \left\{j \varphi^{ \pm}\right\}$. The linearized field equation in the time domain reads

$$
\frac{1}{v_{g}} \frac{\partial}{\partial t}\left(\frac{\delta a^{ \pm}}{a_{s}^{ \pm}}+j \delta \varphi^{ \pm}\right)+j \delta k \pm \frac{\partial}{\partial z}\left(\frac{\delta a^{ \pm}}{a_{s}^{ \pm}}+j \delta \varphi^{ \pm}\right)=\frac{f^{ \pm}}{u_{s}^{ \pm}}
$$

with the stationary solution $u_{s}^{ \pm}(t, z)=a_{s}^{ \pm} \exp \left\{j \varphi_{s}^{ \pm}\right\}$. Linearization of the carrier equation yields

$$
\frac{\partial}{\partial t} \delta N=\delta J-\frac{\delta N}{\tau_{R}}-\frac{\partial R_{\mathrm{st}}}{\partial S} \delta S+F_{N} .
$$

For the stationary carrier density distribution $N_{s}(z)$, the stationary photon density distribution $S_{s}=S_{s}^{+}(z)+S_{s}^{-}(z)$, and, accordingly, the stationary wavenumber $k_{s}=k\left(\omega_{s}, N_{s}, S_{s}\right)$, the differentials in (10) are given as

$$
\begin{aligned}
\delta S & =2\left(S_{s}^{+} \frac{\delta a^{+}}{a_{s}^{+}}+S_{s}^{-} \frac{\delta a^{-}}{a_{s}^{-}}\right) \\
\delta N & =N-N_{s} \\
\delta k & =k-k_{s}=\frac{\partial k}{\partial N} \delta N+\frac{\partial k}{\partial S} \delta S
\end{aligned}
$$

while $\tau_{R}=\left[(\partial / \partial N)\left(R_{\mathrm{sp}}+R_{\mathrm{st}}+R_{\mathrm{ASE}}\right)\right]^{-1}$ is the local carrier lifetime.

In a notation similar to that used for the field envelopes $u^{ \pm}(t, z)$, the incident fields just outside the facets are written as $v^{ \pm}(t)=b^{ \pm}(t) \exp \left\{j \theta^{ \pm}(t)\right\}$, where $b^{ \pm}(t)$ and $\theta^{ \pm}(t)$ are real functions. The boundary conditions for the small-signal fields are found by linearizing (3). For later use, we separate into real and imaginary parts. For $z=0$, the linearized boundary conditions become

$$
\begin{aligned}
\frac{\delta a^{+}}{a_{s}^{+}} & =\left(\frac{\delta a^{-}}{a_{s}^{-}}\right) \gamma_{r}^{+}-\delta \varphi^{-} \gamma_{i}^{+}+\left(\boldsymbol{v}_{0}\right)_{1} \\
\delta \varphi^{+} & =\left(\frac{\delta a^{-}}{a_{s}^{-}}\right) \gamma_{i}^{+}+\delta \varphi^{-} \gamma_{r}^{+}+\left(\boldsymbol{v}_{0}\right)_{2} .
\end{aligned}
$$

Similarly, for $z=l$, we have

$$
\begin{aligned}
\frac{\delta a^{-}}{a_{s}^{-}} & =\left(\frac{\delta a^{+}}{a_{s}^{+}}\right) \gamma_{r}^{-}-\delta \varphi^{+} \gamma_{i}^{-}+\left(\boldsymbol{v}_{0}\right)_{3} \\
\delta \varphi^{-} & =\left(\frac{\delta a^{+}}{a_{s}^{+}}\right) \gamma_{i}^{-}+\delta \varphi^{+} \gamma_{r}^{-}+\left(\boldsymbol{v}_{0}\right)_{4} .
\end{aligned}
$$

The coefficients $\gamma_{r}^{ \pm}$and $\gamma_{i}^{ \pm}$are real numbers defined through

$$
\begin{aligned}
\gamma_{r}^{+}+j \gamma_{i}^{+} & =1-\frac{t_{l r} v_{s}^{+}}{u_{s}^{+}(0)} \\
& =r_{1} \frac{u_{s}^{-}(0)}{u_{s}^{+}(0)} \\
\gamma_{r}^{-}+j \gamma_{i}^{-} & =1-\frac{t_{r l} v_{s}^{-} e^{-j k_{r} l}}{u_{s}^{-}(l)} \\
& =r_{2} e^{-2 j k_{r} l} \frac{u_{s}^{+}(l)}{u_{s}^{-}(l)} .
\end{aligned}
$$

The stationary incident fields $v_{s}^{ \pm}$are defined as $v_{s}^{ \pm}=$ $b_{s}^{ \pm} \exp \left\{j \theta_{s}^{ \pm}\right\}$. The four-dimensional vector $\boldsymbol{v}_{0}$, which has real components in the time domain, is for later use defined in the Laplace domain

$$
\begin{aligned}
& \left(\widetilde{\boldsymbol{v}}_{0}(s)\right)_{1}+j\left(\widetilde{\boldsymbol{v}}_{0}(s)\right)_{2}=\rho_{L} \widetilde{\delta v^{+}}(s) \\
& \left(\widetilde{\boldsymbol{v}}_{0}(s)\right)_{3}+j\left(\widetilde{\boldsymbol{v}}_{0}(s)\right)_{4}=\rho_{R} \widetilde{\delta v^{-}}(s)
\end{aligned}
$$

where $\left(\tilde{\boldsymbol{v}}_{0}\right)_{i}$ denotes the $i$ th component of $\tilde{\boldsymbol{v}}_{0}$ and $\rho_{R}$ and $\rho_{L}$ are given as

$$
\begin{aligned}
\rho_{L} & =\frac{t_{l r}}{u_{s}^{+}(0)} \\
\rho_{R} & =\frac{t_{r l} e^{-j k_{r} l}}{u_{s}^{-}(l)} .
\end{aligned}
$$

A tilde above symbols indicates a function in the Laplace domain and the Laplace variable $s$ is related to the baseband frequency $\Omega$ by $s=j \Omega$. The differential of $v^{ \pm}$is seen to be

$$
\widetilde{\delta v^{ \pm}}=\widetilde{\delta b^{ \pm}} e^{j \theta_{s}^{ \pm}}+b_{s}^{ \pm} e^{j \theta_{s}^{ \pm}} \widetilde{j \delta \theta^{ \pm}} .
$$

Next, the linearized field (9) is separated into real and imaginary parts and then Laplace transformed. Also, the linearized carrier (10) is Laplace transformed and the resulting $\widetilde{\delta N}(s, z)$ is inserted in the Laplace transformed linearized field (9). The Laplace transformed small-signal fields are then gathered in a vector $\boldsymbol{x}=\left(\widetilde{\delta a^{+}} / a_{s}^{+}, \widetilde{\delta \varphi^{+}}, \widetilde{\delta a^{-}} / a_{s}^{-}, \widetilde{\delta \varphi^{-}}\right)^{T}$, where the superscript " $\mathrm{T}$ " is the transpose. The components of $\boldsymbol{x}$ then depend on $s$ and $z$. A matrix equation for $\boldsymbol{x}$ can accordingly be written as

$$
\left(\boldsymbol{M}_{0}+\boldsymbol{M}_{1} \frac{\partial}{\partial z}\right) \boldsymbol{x}=\widetilde{\delta J} \boldsymbol{h}_{N}+\boldsymbol{f}
$$


The $4 \times 4$ matrices $\boldsymbol{M}_{0}(s, z)$ and $\boldsymbol{M}_{1}(z)$ along with the 4-D vectors $\boldsymbol{h}_{N}$ and $\boldsymbol{f}$ are given in Appendix B. $\boldsymbol{f}$ is the Langevin noise vector function.

With unit vectors $\boldsymbol{e}_{i}$ having components $\left(\boldsymbol{e}_{i}\right)_{j}=\delta_{i j}, i=$ $1, \ldots, 4$, the boundary conditions for the small-signal fields in (12) and (13) may also be stated as

$$
\begin{aligned}
\left(\boldsymbol{e}_{1}^{T}-\gamma_{r}^{+} \boldsymbol{e}_{3}^{T}+\gamma_{i}^{+} \boldsymbol{e}_{4}^{T}\right) \boldsymbol{x}(s, 0) & =\left(\widetilde{\boldsymbol{v}}_{0}(s)\right)_{1} \\
\left(\boldsymbol{e}_{2}^{T}-\gamma_{i}^{+} \boldsymbol{e}_{3}^{T}-\gamma_{r}^{+} \boldsymbol{e}_{4}^{T}\right) \boldsymbol{x}(s, 0) & =\left(\widetilde{\boldsymbol{v}}_{0}(s)\right)_{2} \\
\left(\boldsymbol{e}_{3}^{T}-\gamma_{r}^{-} \boldsymbol{e}_{1}^{T}+\gamma_{i}^{-} \boldsymbol{e}_{2}^{T}\right) \boldsymbol{x}(s, l) & =\left(\widetilde{\boldsymbol{v}}_{0}(s)\right)_{3} \\
\left(\boldsymbol{e}_{4}^{T}-\gamma_{i}^{-} \boldsymbol{e}_{1}^{T}-\gamma_{r}^{-} \boldsymbol{e}_{2}^{T}\right) \boldsymbol{x}(s, l) & =\left(\widetilde{\boldsymbol{v}}_{0}(s)\right)_{4} .
\end{aligned}
$$

The differential operator $\boldsymbol{M}_{0}+\boldsymbol{M}_{1}(\partial / \partial z)$ in (18) has the adjoint operator $\boldsymbol{M}_{0}^{\dagger}-\boldsymbol{M}_{1}^{\dagger}(\partial / \partial z)$, where the superscript " $\dagger$ " indicates a Hermitian conjugation. The four-component Green's vector functions $\boldsymbol{\zeta}_{i}\left(s, z^{\prime}, z\right), i=1, \ldots, 4$, for the adjoint differential operator are now defined as solutions to the equation

$$
\left(\boldsymbol{M}_{0}^{\dagger}\left(s, z^{\prime}\right)-\boldsymbol{M}_{1}^{\dagger}\left(z^{\prime}\right) \frac{\partial}{\partial z}\right) \boldsymbol{\zeta}_{i}\left(s, z^{\prime}, z\right)=\boldsymbol{e}_{i} \delta\left(z^{\prime}-z\right)
$$

In order to obtain an explicit expression for $\boldsymbol{x}(s, z), \boldsymbol{\zeta}_{i}$ has to obey the following boundary conditions for $z=0$ and $z=l$ :

$$
\begin{aligned}
\left(\boldsymbol{e}_{1}^{T} \gamma_{r}^{+}+\boldsymbol{e}_{2}^{T} \gamma_{i}^{+}+\boldsymbol{e}_{3}\right) \boldsymbol{M}_{1}^{\dagger}(0) \boldsymbol{\zeta}_{i}(s, 0, z) & =0 \\
\left(-\boldsymbol{e}_{1}^{T} \gamma_{i}^{+}+\boldsymbol{e}_{2}^{T} \gamma_{r}^{+}+\boldsymbol{e}_{4}\right) \boldsymbol{M}_{1}^{\dagger}(0) \boldsymbol{\zeta}_{i}(s, 0, z) & =0 \\
\left(\boldsymbol{e}_{1}^{T}+\boldsymbol{e}_{3}^{T} \gamma_{r}^{-}+\boldsymbol{e}_{4}^{T} \gamma_{i}^{-}\right) \boldsymbol{M}_{1}^{\dagger}(l) \zeta_{i}(s, l, z) & =0 \\
\left(\boldsymbol{e}_{2}^{T}-\boldsymbol{e}_{3}^{T} \gamma_{i}^{-}+\boldsymbol{e}_{4}^{T} \gamma_{r}^{-}\right) \boldsymbol{M}_{1}^{\dagger}(l) \zeta_{i}(s, l, z) & =0 .
\end{aligned}
$$

It is further shown in Appendix $\mathrm{C}$ that, by calculating $\zeta_{i}$, the vector $\boldsymbol{x}(s, z)$ can be found explicitly through

$$
\begin{aligned}
(\boldsymbol{x}(s, z))_{i} \\
=\int_{0}^{l} \boldsymbol{\zeta}_{i}^{\dagger}\left(s, z^{\prime}, z\right)\left[\widetilde{\delta J}\left(s, z^{\prime}\right) \boldsymbol{h}_{N}\left(s, z^{\prime}\right)+\boldsymbol{f}\left(s, z^{\prime}\right)\right] d z^{\prime} \\
\quad+\left(\left[\boldsymbol{M}_{1}^{\dagger}(0) \boldsymbol{\zeta}_{i}(s, 0, z)\right]^{\dagger}\right)_{1}\left(\widetilde{\boldsymbol{v}}_{0}(s)\right)_{1} \\
\quad+\left(\left[\boldsymbol{M}_{1}^{\dagger}(0) \boldsymbol{\zeta}_{i}(s, 0, z)\right]^{\dagger}\right)_{2}\left(\widetilde{\boldsymbol{v}}_{0}(s)\right)_{2} \\
\quad-\left(\left[\boldsymbol{M}_{1}^{\dagger}(l) \boldsymbol{\zeta}_{i}(s, l, z)\right]^{\dagger}\right)_{3}\left(\widetilde{\boldsymbol{v}}_{0}(s)\right)_{3} \\
\quad-\left(\left[\boldsymbol{M}_{1}^{\dagger}(l) \boldsymbol{\zeta}_{i}(s, l, z)\right]^{\dagger}\right)_{4}\left(\widetilde{\boldsymbol{v}}_{0}(s)\right)_{4} .
\end{aligned}
$$

With (22), we can calculate $\boldsymbol{x}(s, z)$ for either a pump rate modulation $\widetilde{\delta J}(s, z)$, an optical modulation through $\widetilde{\boldsymbol{v}}_{0}(s)$, or for a nonzero Langevin noise vector $\boldsymbol{f}(s, z)$. It requires only that $\zeta_{i}\left(s, z^{\prime}, z\right)$ be calculated.

As in [19], we only evaluate $x$ at one of the end facets where the output is measured in an experiment. Here we choose $z=l$, i.e., at the right facet. In this limit, we use the shorthand notation $\zeta_{i}(s, z) \equiv \zeta_{i}\left(s, z, l^{-}\right)$for $z<l$ and the limit of $\zeta_{i}(s, z)$ for $z \rightarrow l^{-}$is denoted $\zeta_{i}(s, l)$. In this limit, (20) is a homogeneous equation. Defining vectors $\boldsymbol{y}_{i}(s, z)=\boldsymbol{M}_{1}^{\dagger}(z) \boldsymbol{\zeta}_{i}(s, z)$ and the matrix $\boldsymbol{M}(s, z)=\left[\boldsymbol{M}_{1}^{-1}(z) \boldsymbol{M}_{0}(s, z)\right]^{\dagger}$ we get the homogeneous equation for $\boldsymbol{y}_{i}$ from (20)

$$
\frac{\partial}{\partial z} \boldsymbol{y}_{i}=\boldsymbol{M}(s, z) \boldsymbol{y}_{i}(s, z) .
$$

The boundary conditions for $\boldsymbol{y}_{i}$ given in (21) become

$$
\begin{aligned}
& \left(\boldsymbol{e}_{1}^{T}+\boldsymbol{e}_{3}^{T} \gamma_{r}^{-}+\boldsymbol{e}_{4}^{T} \gamma_{i}^{-}\right) \boldsymbol{y}_{i}(s, l) \\
& =\left(\boldsymbol{e}_{1}^{T}+\boldsymbol{e}_{3}^{T} \gamma_{r}^{-}+\boldsymbol{e}_{4}^{T} \gamma_{i}^{-}\right) \boldsymbol{e}_{i} \\
& \left(\boldsymbol{e}_{2}^{T}-\boldsymbol{e}_{3}^{T} \gamma_{i}^{-}+\boldsymbol{e}_{4}^{T} \gamma_{r}^{-}\right) \boldsymbol{y}_{i}(s, l) \\
& =\left(\boldsymbol{e}_{2}^{T}-\boldsymbol{e}_{3}^{T} \gamma_{i}^{-}+\boldsymbol{e}_{4}^{T} \gamma_{r}^{-}\right) \boldsymbol{e}_{i} \\
& \left(\boldsymbol{e}_{1}^{T} \gamma_{r}^{+}+\boldsymbol{e}_{2}^{T} \gamma_{i}^{+}+\boldsymbol{e}_{3}\right) \boldsymbol{y}_{i}(s, 0) \\
& \quad=0 \\
& \left(-\boldsymbol{e}_{1}^{T} \gamma_{i}^{+}+\boldsymbol{e}_{2}^{T} \gamma_{r}^{+}+\boldsymbol{e}_{4}\right) \boldsymbol{y}_{i}(s, 0) \\
& \quad=0 .
\end{aligned}
$$

The vector function (23) with boundary conditions in (24) can then be solved, as described in the remainder of this section, to obtain $\boldsymbol{y}_{i}$ and in turn the Green's functions $\boldsymbol{\zeta}_{i}(s, z)$.

Let $\overline{\boldsymbol{y}}_{j}, j=1, \ldots, 4$, be four linearly independent solutions to (23). Then, $\boldsymbol{y}_{i}$ may be written as

$$
\boldsymbol{y}_{i}(s, z)=\sum_{j} a_{i j}(s) \overline{\boldsymbol{y}}_{j}(s, z) .
$$

At the left boundary, $\overline{\boldsymbol{y}}_{j}$ is chosen to satisfy the condition

$$
\overline{\boldsymbol{y}}_{j}(s, 0)=\boldsymbol{e}_{j}
$$

for $j=1, \ldots, 4$. By starting at $z=0$ with the conditions (26), the functions $\bar{y}_{j}$ are calculated numerically by propagation to $z=l$ by the operator $\partial / \partial z-\boldsymbol{M}(s, z)$. Next, define the matrix $\boldsymbol{Y}$ with column vectors $\overline{\boldsymbol{y}}_{i}$ by

$$
\boldsymbol{Y}=\left\{\overline{\boldsymbol{y}}_{1}, \overline{\boldsymbol{y}}_{2}, \overline{\boldsymbol{y}}_{3}, \overline{\boldsymbol{y}}_{4}\right\}
$$

and the vectors $\boldsymbol{a}_{i}(s)$ with components

$$
\left(\boldsymbol{a}_{i}\right)_{j}=a_{i j} .
$$

Calculating $(\boldsymbol{x}(s, l))_{i}$ for $i=1,2$ gives the small-signal deviations at the right facet. Then, we need to obtain $\boldsymbol{\zeta}_{i}$ and hence $\boldsymbol{y}_{i}$ for $i=1,2$ only. From the boundary conditions (24), vectors $\boldsymbol{a}_{i}$ are obtained as

$$
\boldsymbol{a}_{i}(s)=Q^{-1}(s) \boldsymbol{e}_{i}, \quad i=1,2
$$

where $\boldsymbol{Q}$ is the matrix

$$
\boldsymbol{Q}(s)=\left\{\begin{array}{c}
\left(\boldsymbol{e}_{1}^{T}+\gamma_{r}^{-} \boldsymbol{e}_{3}^{T}+\gamma_{i}^{-} \boldsymbol{e}_{4}^{T}\right) \boldsymbol{Y}(s, l) \\
\left(\boldsymbol{e}_{2}^{T}-\gamma_{i}^{-} \boldsymbol{e}_{3}^{T}+\gamma_{r}^{-} \boldsymbol{e}_{4}^{T}\right) \boldsymbol{Y}(s, l) \\
\left(\gamma_{r}^{+} \boldsymbol{e}_{1}^{T}+\gamma_{i}^{+} \boldsymbol{e}_{2}^{T}+\boldsymbol{e}_{3}^{T}\right) \boldsymbol{Y}(s, 0) \\
\left(-\gamma_{i}^{+} \boldsymbol{e}_{1}^{T}+\gamma_{r}^{+} \boldsymbol{e}_{2}^{T}+\boldsymbol{e}_{4}^{T}\right) \boldsymbol{Y}(s, 0)
\end{array}\right\} .
$$

Having obtained $\boldsymbol{a}_{i}$ and $\overline{\boldsymbol{y}}_{i}(s, z)$, we may calculate $\boldsymbol{y}_{i}(s, z)$ from (25). The Green's functions $\zeta_{i}(s, z)$ to be used when obtaining $(\boldsymbol{x})_{i}$ in (22) are then found using $\boldsymbol{\zeta}_{i}(s, z)=\left(\boldsymbol{M}_{1}^{\dagger}\right)^{-1} \boldsymbol{y}_{i}(s, z)$.

In the following, we only consider input from the left facet, i.e., $v^{-}(t)=0$ and hence $\left(\boldsymbol{v}_{0}\right)_{3}=\left(\boldsymbol{v}_{0}\right)_{4}=0$. It should be noted 
that devices with multiple sections separated by reflective elements and/or with grating structures can be treated by following [19]. Here we have focused on waveguides without internal reflections or grating sections. Notably, however, the pump rate $\widetilde{J}(s, z)$ can be varied along the $z$-axis.

\section{EXPRESSIONS FOR MODULATION RESPONSES}

By calculating $\boldsymbol{y}_{i}$ and hence $\zeta_{i}$, we can obtain expressions for small-signal modulation responses at the right facet. Responses caused by either optical modulation of the incident field or by pump rate modulation may be obtained from (22) with $z=l$ and $\boldsymbol{f}=0$. Since the responses are calculated in the Laplace domain, they are in general complex giving both a magnitude part and an argument.

\section{A. Responses Due to Optical Modulation of Incident Field}

The general procedure for deriving expressions for responses due to optical modulation of the incident field is described in the following. In (22) set $\widetilde{\delta J}(s, z)=0$ and $\boldsymbol{f}=0$. The modulation format can be either an amplitude modulation $\widetilde{\delta b^{+}}(s)$, a phase modulation $\widetilde{\delta \theta^{+}}(s)$, or a frequency modulation $(s / 2 \pi) \widetilde{\delta \theta^{+}}(s)$. Then, from (15) and (17), expressions for $\left(\widetilde{\boldsymbol{v}}_{0}\right)_{1}$ and $\left(\widetilde{\boldsymbol{v}}_{0}\right)_{2}$ in terms of $\widetilde{\delta b^{+}}$(for amplitude modulation) or $\widetilde{\delta \theta^{+}}$(for phase or frequency modulation) can be found. The response can be an amplitude response, a phase response, or a frequency response.

The output power $P_{R}$ from the right facet is proportional to $\left(a^{+}\right)^{2}$ at $z=l$ and the power response follows as

$$
\delta P_{R}(s)=2 P_{R} \frac{\delta a^{+}(s, l)}{a_{s}^{+}(l)}=2 P_{R}(\boldsymbol{x}(s, l))_{1} .
$$

In the case of optical amplitude modulation, $\widetilde{\delta b^{+}}(s)$, we have $\widetilde{\delta \theta^{+}}(s)=0$. Moreover, it is usually more practical to speak in terms of a modulation of the optical power. Using (8a), the optical power modulation may be written as

$$
\delta P_{\text {in }}^{+}(s)=2 \sqrt{2 \epsilon_{0} c P_{\text {in }}^{+}} \widetilde{\delta b^{+}}(s) .
$$

The output power response due to power modulation of the input signal outside the left facet follows from (15), (16), (17), (22), (31), and (32) as

$$
\begin{aligned}
\frac{\delta P_{R}}{\delta P_{\text {in }}^{+}}(s)=\frac{P_{R}}{\sqrt{P_{\text {in }}^{+}}} \frac{1}{\sqrt{2 \epsilon_{0} c}} & {\left[\left(\boldsymbol{y}_{1}^{*}(s, 0)\right)_{1} \operatorname{Re}\left\{\frac{t_{l r} e^{j \theta_{s}^{+}}}{u_{s}^{+}(0)}\right\}\right.} \\
& \left.+\left(\boldsymbol{y}_{1}^{*}(s, 0)\right)_{2} \operatorname{Im}\left\{\frac{t_{l r} e^{j \theta_{s}^{+}}}{u_{s}^{+}(0)}\right\}\right] .
\end{aligned}
$$

The magnitude of (33) then gives the amplification while the argument yields the phase change of the incident RF-envelope signal at the output as compared with the input. The latter, the $\mathrm{RF}$-phase change, is used as a measure of slow/fast light in microwave photonics.
Similarly, we find that the optical phase response due to an optical power modulation of the incident signal, i.e., how $\varphi^{+}$ changes at the right facet when $P_{\text {in }}^{+}$is varied, in units [rad/W] becomes

$$
\begin{aligned}
\frac{\delta \varphi^{+}}{\delta P_{\text {in }}^{+}}(s)=\frac{1}{2 \sqrt{P_{\text {in }}^{+}}} \frac{1}{\sqrt{2 \epsilon_{0} c}} & {\left[\left(\boldsymbol{y}_{2}^{*}(s, 0)\right)_{1} \operatorname{Re}\left\{\frac{t_{l r} e^{j \theta_{s}^{+}}}{u_{s}^{+}(0)}\right\}\right.} \\
& \left.+\left(\boldsymbol{y}_{2}^{*}(s, 0)\right)_{2} \operatorname{Im}\left\{\frac{t_{l r} e^{j \theta_{s}^{+}}}{u_{s}^{+}(0)}\right\}\right] .
\end{aligned}
$$

In the case of an optical phase modulation $\widetilde{\delta \theta^{+}}$of the incident field, then $\widehat{\delta b^{+}}=0$. The power response due to an optical phase modulation outside the left facet becomes

$$
\begin{aligned}
\frac{\delta P_{R}}{\delta \theta^{+}}(s)=2 P_{R} \sqrt{\frac{P_{\text {in }}^{+}}{2 \epsilon_{0} c}}[ & \left(\boldsymbol{y}_{1}^{*}(s, 0)\right)_{2} \operatorname{Re}\left\{\frac{t_{l r} e^{j \theta_{s}^{+}}}{u_{s}^{+}(0)}\right\} \\
& \left.-\left(\boldsymbol{y}_{1}^{*}(s, 0)\right)_{1} \operatorname{Im}\left\{\frac{t_{l r} e^{j \theta_{s}^{+}}}{u_{s}^{+}(0)}\right\}\right]
\end{aligned}
$$

with units [W/rad]. For the case $r_{1}=r_{2}=0$, it is first seen that $\operatorname{Im}\left(t_{l r} e^{j \theta_{s}^{+}} / u_{s}^{+}(0)\right)=0$, and, second, it can be shown that $\left(\boldsymbol{y}_{1}(s, z)\right)_{2}=0$. Then, according to (35), the optical power response to a phase modulation, $\delta P_{R} / \delta \theta^{+}$, is always zero. This is understandable as a phase fluctuation at the input is not "seen" by the semiconductor gain material. However, for finite $r_{1}$ and $r_{2}, \operatorname{Im}\left(t_{l r} e^{j \theta_{s}^{+}} / u_{s}^{+}(0)\right)$ and $\left(\boldsymbol{y}_{1}(s, z)\right)_{2}$ are in general finite and one can have a finite power response $\delta P_{R} / \delta \theta^{+}$.

The optical phase response at the output caused by an optical phase modulation of the incident field is written as

$$
\begin{aligned}
\frac{\delta \varphi^{+}}{\delta \theta^{+}}(s, l)=\sqrt{\frac{P_{\mathrm{in}}^{+}}{2 \epsilon_{0} c}}\left[\left(\boldsymbol{y}_{2}^{*}(s, 0)\right)_{2} \operatorname{Re}\left\{\frac{t_{l r} e^{j \theta_{s}^{+}}}{u_{s}^{+}(0)}\right\}\right. \\
\left.-\left(\boldsymbol{y}_{2}^{*}(s, 0)\right)_{1} \operatorname{Im}\left\{\frac{t_{l r} e^{j \theta_{s}^{+}}}{u_{s}^{+}(0)}\right\}\right] .
\end{aligned}
$$

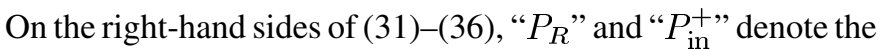
stationary output and input powers, respectively. Again, when $r_{1}=r_{2}=0$, there is no resonant response now implying a unity phase response $\left|\delta \varphi^{+} / \delta \theta^{+}(s)\right|=1$. Nevertheless, finite reflectivities $r_{1}$ and $r_{2}$ will in general lead to magnitudes that can differ from unity.

In measurements of responses, one will often perform a reference measurement to calibrate the experimental setup. For example, when using a network analyzer to measure responses at a range of modulation frequencies, one can do a reference measurement at the transparency point of an SOA, to which other measurements can be compared.

Next, the expressions for responses due a modulation of the current are given from [19]. They are the same for an SOA or a laser subject to optical injection as for a laser without light injection. The modulation current per unit length is given as $\widetilde{\delta I}_{z}(s, z)=e A_{c} \widetilde{\delta J}(s, z)$. When modulating the current over 
TABLE I

LIST OF PARAMETER VALUES

\begin{tabular}{lccll}
\hline Parameter & Symbol & Value & Unit & Ref. \\
\hline Ridge width & $w$ & 2.0 & $\mu \mathrm{m}$ & {$[21]$} \\
Active layer thickness & $h_{a}$ & 29 & $\mathrm{~nm}$ & {$[21]$} \\
Confinement factor & $\Gamma$ & 0.02 & & {$[21]$} \\
Internal loss & $\alpha_{i}$ & 8.5 & $\mathrm{~cm}^{-1}$ & {$[22]$} \\
Recombination coefficient & $\mathrm{A}$ & $1 \cdot 10^{8}$ & $\mathrm{~s}^{-1}$ & {$[20]$} \\
Recombination coefficient & $\mathrm{B}$ & $2.5 \cdot 10^{-17}$ & $\mathrm{~s}^{-1}$ & {$[20]$} \\
Recombination coefficient & $\mathrm{C}$ & $9.4 \cdot 10^{-41}$ & $\mathrm{~s}^{-1}$ & {$[20]$} \\
Nonlinear gain coefficient & $\epsilon$ & $2 \cdot 10^{-23}$ & $\mathrm{~m}^{3}$ & {$[19]$} \\
Linewidth enhancement factor & $\alpha$ & 5.0 & & \\
Effective refractive index & $n_{r}$ & 3.5 & & \\
Effective group index & $n_{g}$ & 4.0 & & \\
Reference wavelength & $\lambda_{r}$ & 1530 & $\mathrm{~nm}$ & \\
\hline
\end{tabular}

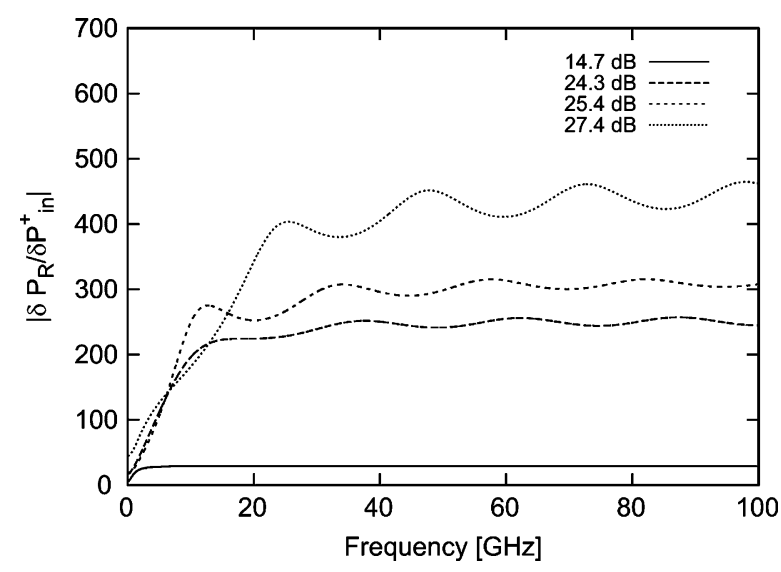

Fig. 2. Magnitude of SOA power response due power modulation of the incident field, see (33), for four different chip gains. The SOA has length $l=$ $1.5 \mathrm{~mm}$ and facet reflectivities $r_{1}^{2}=r_{2}^{2}=10^{-4}$.

the length of the amplifier, the power output power response, phase response, and frequency response are given as

$$
\begin{aligned}
\delta P_{R}(s) & =\frac{2 P_{R}}{e A_{c}} \int_{0}^{l} \boldsymbol{\zeta}_{1}^{\dagger}(s, z) \widetilde{\delta I_{z}}(s, z) \boldsymbol{h}_{N}(s, z) d z \\
\delta \varphi(s) & =\frac{1}{e A_{c}} \int_{0}^{l} \zeta_{2}^{\dagger}(s, z) \widetilde{\delta I_{z}}(s, z) \boldsymbol{h}_{N}(s, z) d z \\
\delta f(s) & =\frac{s}{2 \pi e A_{c}} \int_{0}^{l} \zeta_{2}^{\dagger}(s, z) \widetilde{\delta I_{z}}(s, z) \boldsymbol{h}_{N}(s, z) d z
\end{aligned}
$$

As exemplified in [19] the allowed $z$-dependence of the current modulation can be used for the study of multi-electrode devices. In all examples of calculated responses in this paper we regard one-electrode devices and moreover set $\theta_{s}^{ \pm}=0$. All shown example calculations share the parameter values listed in Table I.

\section{EXAMPLES OF SOA MODULATION RESPONSES}

Figs. 2 and 3 show examples of the magnitude and the argument, respectively, of the power response due to a power modulation of the optical input given in (33) for an SOA with end-facet reflectivities $r_{1}^{2}=r_{2}^{2}=10^{-4}$. The stationary input power is fixed at $P_{\mathrm{in}}^{+}=0.3 \mathrm{~mW}$ while the pump rate is varied giving different chip gains. Here, the chip gain is defined as

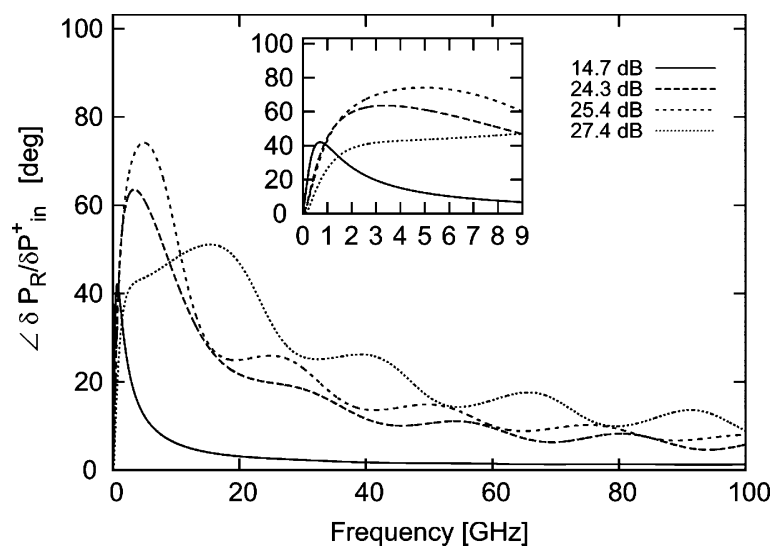

Fig. 3. Argument of SOA power response due to power modulation of the incident field, see (33). Same cases as in Fig. 2. Inset shows close-up for lower frequencies.

$P_{R} / P_{\mathrm{in}}^{+}$. For low values of the chip gain (when the amplifier is unsaturated), one sees the expected characteristics in the highand low-frequency limits of the magnitude in Fig. 2: for low modulation frequencies, the response is inhibited by the carrier-density dynamics of the gain material [11], [23]. In the high-frequency limit, the response approaches the chip gain of the stationary solution since the carrier density cannot follow the rapid fluctuations of the field. With increasing chip gain and thus increasing intra-cavity intensity, the carrier lifetime decreases giving a wider dip in the response for the lower frequencies. Moreover, as the chip gain is increased the response curves become increasingly modulated. The modulation period of $25 \mathrm{GHz}$ corresponds to the spacing between the Fabry-Perot modes of the cavity, i.e., $c /\left(2 n_{g} l\right)$, with $l=1.5 \mathrm{~mm}$. The argument of the response shown in Fig. 3 is the phase shift of the injected RF-envelope signal and can be interpreted as slow or fast light depending on the sign. Positive values yield fast light, as seen in an SOA with positive modal gain [7]. The RF-phase shift here has an optimum of about 74 degrees and is clearly affected by the reflections at high gain values.

Examples of calculated optical phase responses caused by a power modulation of the incident field are displayed in Fig. 4. The finite optical phase responses due to a power modulation occur as intensity fluctuations cause carrier fluctuations which in turn induce fluctuations in optical phase through a nonzero $\alpha$. Finite facet reflectivities in the SOA are found to modify the phase-response curves but are not imperative to have responses of finite magnitude. In contrast, responses caused by fluctuations in the optical phase of the incident signal in an active waveguide with zero reflectivities have the magnitudes $\left|\delta P_{R} / \delta \theta^{+}\right|=0$ and $\left|\delta \varphi^{+} / \delta \theta^{+}\right|=1$, i.e., the phase modulation will neither be amplified/attenuated nor cause fluctuations in power. When the facets have finite values, however, the situation may be different. An incident phase modulated field will travel through the device and be partly reflected at the right facet. The left traveling field being reflected at the left facet will interfere with the injected field and thus cause an intensity fluctuation in the total field. The magnitude and argument of $\delta P_{R} / \delta \theta^{+}$ for different chip gains are shown in Fig. 5(a) and (b), respectively. Similarly, in Fig. 6, the magnitude and argument of the 


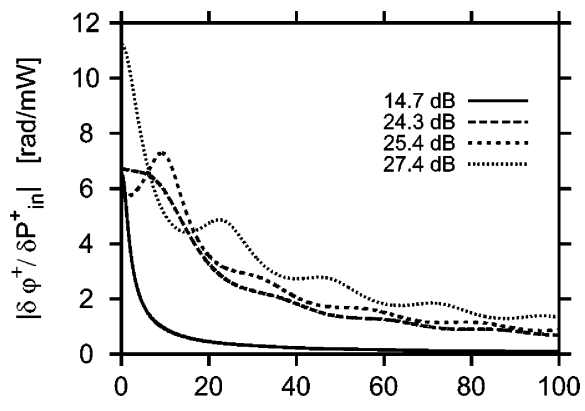

(a)

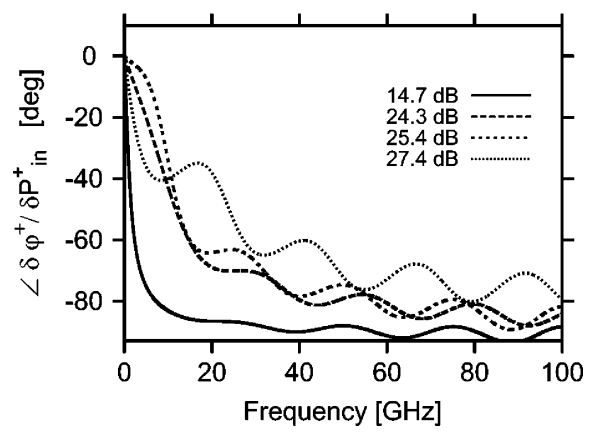

(b)

Fig. 4. SOA optical phase responses due to power modulation of the incident signal calculated from (34). (a) Magnitude. (b) Argument. Same cases as in Fig. 2.

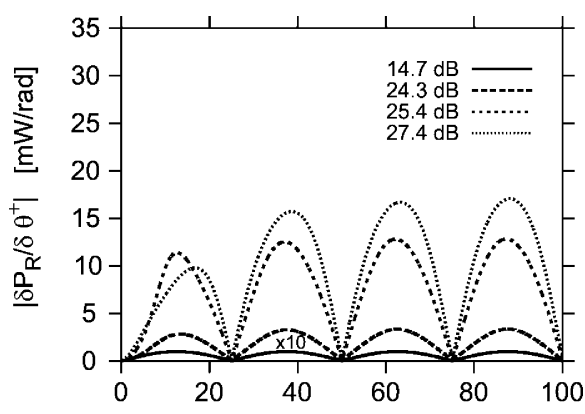

(a)

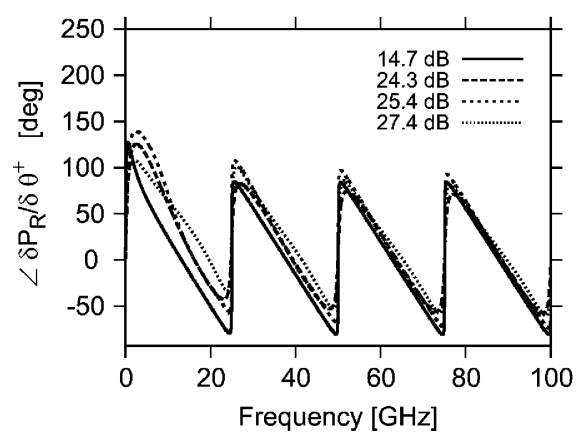

(b)

Fig. 5. (a) Magnitude and (b) argument of the SOA power response due to optical phase modulation calculated from (35). The 14.7-dB plot has been magnified ten times for clarity. Same cases as in Fig. 2.

optical phase response due to a phase modulation $\delta \varphi^{+} / \delta \theta^{+}$can be seen. The magnitude is greater than unity.

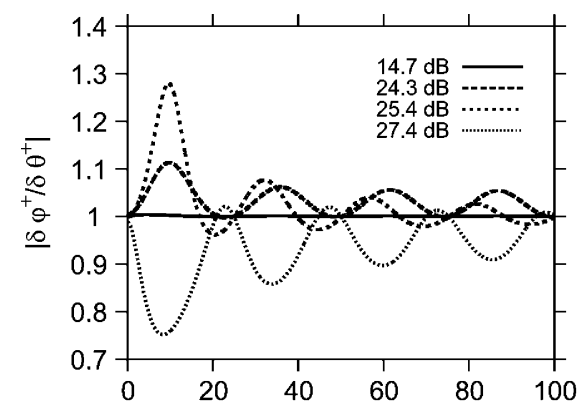

(a)

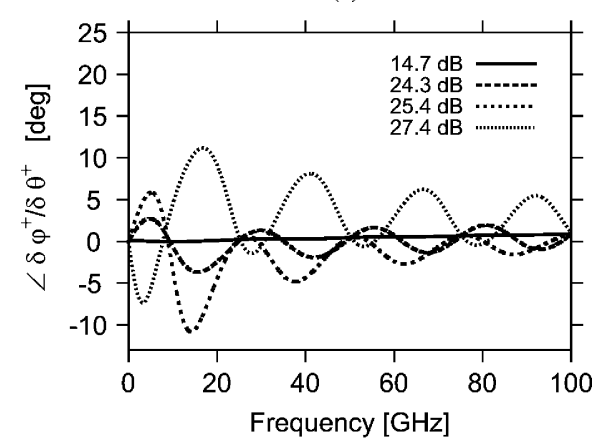

(b)

Fig. 6. (a) Magnitude and (b) argument of the SOA optical phase response due to optical phase modulation calculated from (36). Same cases as in Fig. 2.

\section{EXPRESSIONS FOR NOISE SPECTRA}

Output noise spectra are calculated through (22) with $z=l$ and $\widetilde{\delta J}(s, z)=0$. For this purpose, the diffusion matrix $\boldsymbol{D}$ gives the magnitudes of the Langevin forces in the correlation relation for $\widetilde{\boldsymbol{f}}(s, z)$ as

$$
\left\langle\widetilde{\boldsymbol{f}}(s, z) \tilde{\boldsymbol{f}}^{\dagger}\left(s^{\prime}, z^{\prime}\right)\right\rangle=2 \boldsymbol{D}(s, z) \delta\left(z-z^{\prime}\right) 2 \pi \delta\left(\Omega-\Omega^{\prime}\right)
$$

where " \langle\rangle " denotes an ensemble average. The diffusion matrix $D$ is given as [19]

$$
\begin{aligned}
2 \boldsymbol{D}=\frac{v_{g} g n_{s p}}{2 A_{p h}} \boldsymbol{H} & +2 D_{N N} \boldsymbol{h}_{N} \boldsymbol{h}_{N}^{\dagger} \\
& -\frac{v_{g} n_{s p}}{A_{c}}\left[\boldsymbol{h}_{N}\left(\boldsymbol{e}_{1}+\boldsymbol{e}_{3}\right)^{\dagger}+\left(\boldsymbol{e}_{1}+\boldsymbol{e}_{3}\right) \boldsymbol{h}_{N}^{\dagger}\right] .
\end{aligned}
$$

In (39) the three terms on the r.h.s. stem from spontaneous emission noise, carrier fluctuations, and from the cross-correlation between the carrier fluctuations and the spontaneous emission noise, respectively. The matrix $\boldsymbol{H}$ is diagonal with elements $(\boldsymbol{H})_{11}=(\boldsymbol{H})_{22}=1 / S_{s}^{+}$and $(\boldsymbol{H})_{33}=(\boldsymbol{H})_{44}=1 / S_{s}^{-}$. The diffusion coefficient $D_{N N}$ is stated in Appendix B. The spectral density of two functions $X(t)$ and $Y(t)$ may be written as

$$
\begin{aligned}
S_{X, Y}(\Omega)=\lim _{T \rightarrow \infty} \frac{1}{2 T}\left\langle\widetilde{X}(s) \tilde{Y}^{*}(s)\right\rangle & \\
& \equiv\left\{\widetilde{X}(s) \widetilde{Y}^{*}(s)\right\}_{\mathrm{av}} .
\end{aligned}
$$

Here, $\tilde{X}(s)$ is the Fourier transform of $X(t)$ over a time interval going from $-T$ to $T$.

The output RIN spectrum at the right facet, $\operatorname{RIN}(\Omega)$, is then given by

$$
\operatorname{RIN}(\Omega)=4\left\{\left|(x)_{1}(s, l)\right|^{2}\right\}_{\mathrm{av}} .
$$


Similarly, the output phase noise spectrum $S_{\varphi, \varphi}(\Omega)$ can be written as

$$
S_{\varphi, \varphi}(\Omega)=\left\{\left|(\boldsymbol{x})_{2}(s, l)\right|^{2}\right\}_{\mathrm{av}} .
$$

From (22) and (38), the general cross-correlation spectra involving $\boldsymbol{x}_{i}$ and $\boldsymbol{x}_{j}$ for $i=1,2$ and $j=1,2$ are given as

$$
\begin{aligned}
\left\{(\boldsymbol{x}(s, l))_{i}(\boldsymbol{x}(s, l))_{j}^{*}\right\}_{\mathrm{av}} \\
=\int_{0}^{l} \boldsymbol{\zeta}_{i}^{\dagger}(s, z) 2 \boldsymbol{D}(s, z) \boldsymbol{\zeta}_{j}(s, z) d z \\
\quad+\left\{\left|\left(\widetilde{\boldsymbol{v}}_{0}(s)\right)_{1}\right|^{2}\right\}_{\mathrm{av}}\left(\boldsymbol{y}_{i}(s, 0)\right)_{1}^{*}\left(\boldsymbol{y}_{j}(s, 0)\right)_{1} \\
\quad+\left\{\left|\left(\widetilde{\boldsymbol{v}}_{0}(s)\right)_{2}\right|^{2}\right\}_{\mathrm{av}}\left(\boldsymbol{y}_{i}(s, 0)\right)_{2}^{*}\left(\boldsymbol{y}_{j}(s, 0)\right)_{2} \\
\quad+\left\{\left(\widetilde{\boldsymbol{v}}_{0}(s)\right)_{1}\left(\widetilde{\boldsymbol{v}}_{0}(s)\right)_{2}^{*}\right\}_{\mathrm{av}}\left(\boldsymbol{y}_{i}(s, 0)\right)_{1}^{*}\left(\boldsymbol{y}_{j}(s, 0)\right)_{2} \\
\quad+\left\{\left(\widetilde{\boldsymbol{v}}_{0}(s)\right)_{2}\left(\widetilde{\boldsymbol{v}}_{0}(s)\right)_{1}^{*}\right\}_{\mathrm{av}}\left(\boldsymbol{y}_{i}(s, 0)\right)_{2}^{*}\left(\boldsymbol{y}_{j}(s, 0)\right)_{1} .
\end{aligned}
$$

The terms on the r.h.s. of (43) outside the integral are due to fluctuations on the signal incident on the left facet. For a coherent input signal, i.e., a pure $\mathrm{CW}$ signal, these terms vanish. The autocorrelations involving $\widetilde{\boldsymbol{v}}_{0}$ follow as

$$
\begin{aligned}
\left\{\left|\left(\widetilde{\boldsymbol{v}}_{0}\right)_{1}(s)\right|^{2}\right\}_{\mathrm{av}} & \\
= & \frac{1}{4} \operatorname{RIN}_{v^{+}}(s) \operatorname{Re}^{2}\left\{\rho_{L} v_{s}^{+}\right\}+S_{\delta \theta^{+}, \delta \theta^{+}}(s) \operatorname{Im}^{2}\left\{\rho_{L} v_{s}^{+}\right\} \\
& -2 \operatorname{Re}\left\{S_{\delta b^{+} / b_{s}^{+}, \delta \theta^{+}}(s)\right\} \operatorname{Re}\left\{\rho_{L} v_{s}^{+}\right\} \operatorname{Im}\left\{\rho_{L} v_{s}^{+}\right\}
\end{aligned}
$$

and

$$
\begin{aligned}
& \left\{\left|\left(\widetilde{\boldsymbol{v}}_{0}\right)_{2}(s)\right|^{2}\right\}_{a v} \\
& \quad=\frac{1}{4} \operatorname{RIN}_{v+}(s) \operatorname{Im}^{2}\left\{\rho_{L} v_{s}^{+}\right\}+S_{\delta \theta^{+}, \delta \theta^{+}}(s) \operatorname{Re}^{2}\left\{\rho_{L} v_{s}^{+}\right\} \\
& \quad+2 \operatorname{Re}\left\{S_{\delta b^{+} / b_{s}^{+}, \delta \theta^{+}}(s)\right\} \operatorname{Re}\left\{\rho_{L} v_{s}^{+}\right\} \operatorname{Im}\left\{\rho_{L} v_{s}^{+}\right\}
\end{aligned}
$$

while the cross correlations read

$$
\begin{aligned}
\left\{\left(\widetilde{\boldsymbol{v}}_{0}\right)_{1}(s)\left(\widetilde{\boldsymbol{v}}_{0}\right)_{2}^{*}(s)\right\}_{a v} & \\
= & \frac{1}{4} \operatorname{RIN}_{v^{+}}(s) \operatorname{Re}\left\{\rho_{L} v_{s}^{+}\right\} \operatorname{Im}\left\{\rho_{L} v_{s}^{+}\right\} \\
& -S_{\delta \theta^{+}, \delta \theta^{+}}(s) \operatorname{Re}\left\{\rho_{L} v_{s}^{+}\right\} \operatorname{Im}\left\{\rho_{L} v_{s}^{+}\right\} \\
& +S_{\delta b^{+} / b_{s}^{+}, \delta \theta^{+}}(s) \operatorname{Re}^{2}\left\{\rho_{L} v_{s}^{+}\right\} \\
& -S_{\delta \theta^{+}, \delta b^{+} / b_{s}^{+}}(s) \operatorname{Im}^{2}\left\{\rho_{L} v_{s}^{+}\right\} .
\end{aligned}
$$

Here, $\operatorname{RIN}_{v^{+}}(s)$ is the RIN of the incident signal given by

$$
\operatorname{RIN}_{v^{+}}(\Omega)=4\left\{\left|\frac{\widetilde{\delta b^{+}}(s)}{b_{s}^{+}}\right|^{2}\right\}_{\mathrm{av}} .
$$

Further, $S_{\delta \theta^{+}, \delta \theta^{+}}$is the input phase noise spectrum

$$
S_{\delta \theta^{+}, \delta \theta^{+}}(\Omega)=\left\{\left|\widetilde{\delta \theta^{+}}(s)\right|^{2}\right\}_{\mathrm{av}}
$$

and $S_{\delta b^{+} / b_{s}^{+}, \delta \theta^{+}}$is the input cross-correlation spectrum

$$
S_{\delta b^{+} / b_{s}^{+}, \delta \theta^{+}}(\Omega)=\left\{\frac{\widetilde{\delta b^{+}}(s)}{b_{s}^{+}}\left(\widetilde{\delta \theta^{+}}(s)\right)^{*}\right\}_{\mathrm{av}} .
$$

As previously mentioned in Section IV, for the case of perfectly AR-coated facets $\left(r_{1}=r_{2}=0\right)$ we have $\left(\boldsymbol{y}_{1}(s, z)\right)_{2}=0$ and $\operatorname{Im}\left(\rho_{L} v_{s}^{+}\right)=0$. In this case, e.g., the RIN simplifies to

$$
\begin{aligned}
\operatorname{RIN}(\Omega)=4 \int_{0}^{l} \boldsymbol{\zeta}_{1}^{\dagger}(s, z) 2 \boldsymbol{D}(s, z) \boldsymbol{\zeta}_{1}(s, z) d z & \\
& +\operatorname{RIN}_{v^{+}}(s)\left|\left(\boldsymbol{y}_{1}(s, 0)\right)_{1}\right|^{2} .
\end{aligned}
$$

In (50), the first term is due to the intensity noise of the device while the second term represents the RIN of the input signal being transferred to the right facet by a function depending on the baseband frequency in agreement with [11]. In correspondence with the discussion regarding the power response due to a phase modulation, the input phase-noise spectrum does not enter in (50) because the nonlinearity of the gain material does not depend on the phase of light.

The field power spectrum is the Fourier transform of the field autocorrelation function

$$
S_{E, E}(\Omega)=\int_{-\infty}^{\infty}\left\langle E^{*}(t, l) E(t+\tau, l)\right\rangle e^{-j \Omega \tau} d \tau .
$$

We use the following approximate expression for $S_{E, E}(\Omega)$ (see [24] and [25]):

$$
\begin{aligned}
\frac{S_{E, E}(\Omega)}{\left|u_{s}^{+}(l)\right|^{2}} \simeq & L(\Omega)+\left[\frac{S_{\dot{\varphi}, \dot{\varphi}}(\Omega)-2 \pi \Delta \nu}{\Omega^{2}}\right. \\
& +2 \operatorname{Im}\left\{S_{\delta a^{+} / a_{s}^{+}, \varphi}(\Omega)\right\} \\
& \left.+\frac{1}{4} \operatorname{RIN}(\Omega)\right] \otimes L(\Omega) .
\end{aligned}
$$

The symbol " $\otimes$ " means convolution in the frequency domain, and the function $L(\Omega)$ is the Lorentzian

$$
L(\Omega)=\frac{2 \pi \Delta \nu}{\Omega^{2}+(\pi \Delta \nu)^{2}} .
$$

For a solitary laser without an optical input, the amplitude phase cross-spectral density $S_{\delta a^{+} / a_{s}^{+}, \varphi}(\Omega)$ diverges for $\Omega \rightarrow 0$. However, this is not the case when an optical input is present.

Furthermore $S_{\dot{\varphi}, \dot{\varphi}}$ is the frequency spectrum

$$
\left.S_{\dot{\varphi}, \dot{\varphi}}(\Omega)=\left.\Omega^{2}\left\{\mid(\boldsymbol{x}(s, l))_{2}\right)\right|^{2}\right\}_{\mathrm{av}}
$$

whose low frequency limit gives the spectral linewidth $\Delta \nu=$ $S_{\dot{\varphi}, \dot{\varphi}}(0) / 2 \pi$.

In the case of zero linewidth, the field power spectrum of the output signal becomes

$$
\begin{aligned}
\frac{S_{E, E}(\Omega)}{\left|u_{s}^{+}(l)\right|^{2}} \simeq \delta(\Omega)+ & {\left[S_{\varphi, \varphi}(\Omega)\right.} \\
+ & \left.2 \operatorname{Im}\left\{S_{\delta a^{+} / a_{s}^{+}, \varphi}(\Omega)\right\}+\frac{1}{4} R I N(\Omega)\right]
\end{aligned}
$$

where the Dirac delta function on the r.h.s. is due to the static part of the field.

\section{EXAMPLES OF SOA NOISE SPECTRA}

In the examples of noise spectra for an SOA we assume the input signal to be a noiseless CW signal. Maintaining a fixed 


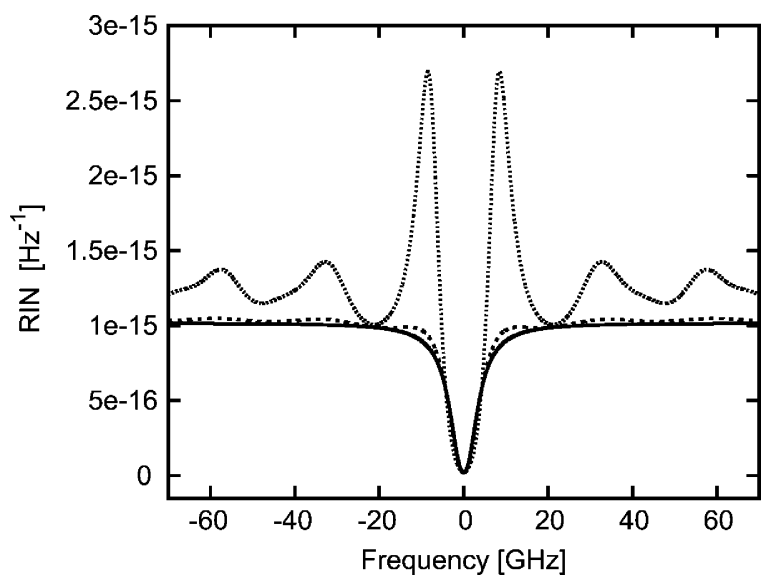

Fig. 7. SOA RIN calculated for different end-facet reflectivities when the chip gain equals $20 \mathrm{~dB}$. Full line $r_{1}^{2}=r_{2}^{2}=0$, dashed line $r_{1}^{2}=r_{2}^{2}=10^{-4}$, and dotted line $r_{1}^{2}=r_{2}^{2}=10^{-3}$.

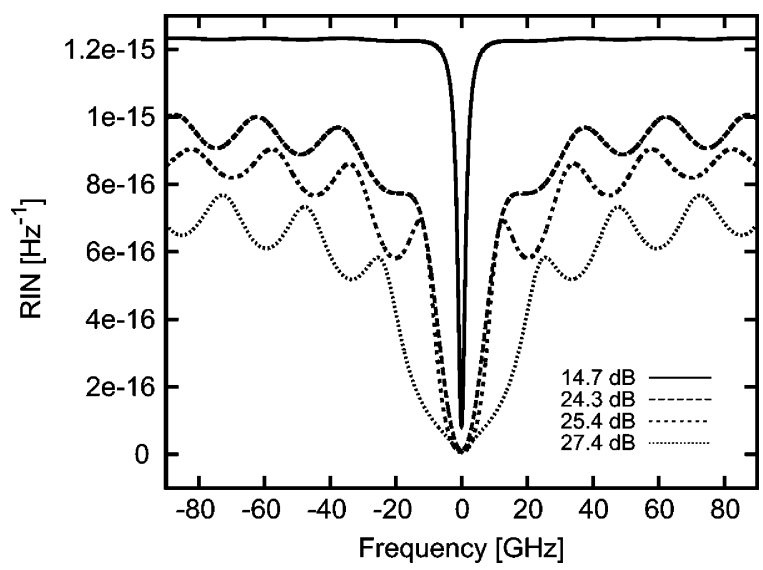

Fig. 8. SOA RIN for $r_{1}^{2}=r_{2}^{2}=10^{-4}$ and $l=1.5 \mathrm{~mm}$. RIN is symmetric in baseband frequency but is for clarity shown for both positive and negative frequencies.

chip gain of $20 \mathrm{~dB}$ and a fixed input power $P_{\mathrm{in}}^{+}=0.3 \mathrm{~mW}$, the RIN spectra in Fig. 7 reveal a growing ripple when increasing values of the end-facet reflectivities of the 1.5 -mm-long device. Cases with power reflectivities $10^{-5}$ and $10^{-6}$ (neither shown) give RINs practically identical to the case of $r_{1}^{2}=r_{2}^{2}=0$.

The RIN spectra shown in Fig. 8 where $r_{1}^{2}=r_{2}^{2}=10^{-4}$ clearly show how signatures of the Fabry-Perot cavity become pronounced as the chip gain is increased for a fixed input power. Four different cases for different values of chip gain are shown. When increasing the chip gain, the level of the RIN becomes lower while the dips, caused by the nonlinear response of the SOA [11], around $\Omega=0$ become shallower and wider. The former effect is due to the increased intensity while the broadened dip is caused by the shortening of the carrier lifetime, in turn also caused by the increasing intensity following the increasing gain. The bandwidth over which the gain material can suppress the intensity fluctuations is governed by the reciprocal carrier lifetime.

Phase noise in an SOA is, in contrast to RIN, enhanced at lower frequencies since the carrier density fluctuations inhibiting intensity noise, cause phase fluctuations through a

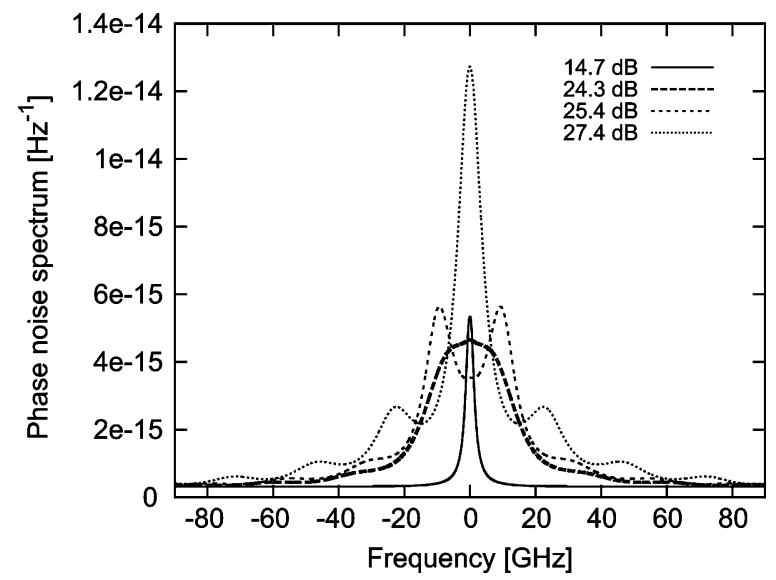

Fig. 9. SOA phase noise spectra for $r_{1}^{2}=r_{2}^{2}=10^{-4}$ and $l=1.5 \mathrm{~mm}$. The phase noise spectrum is symmetric in baseband frequency but is for clarity shown for both positive and negative frequencies.

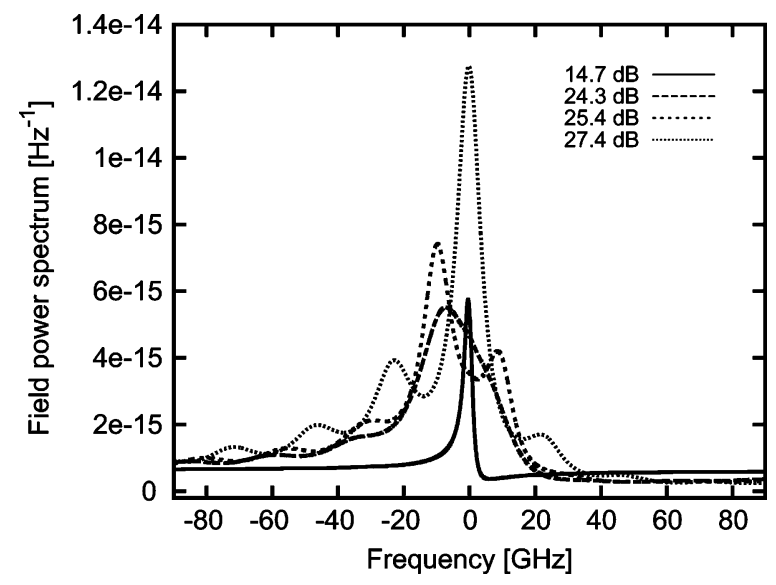

Fig. 10. SOA field power spectra for $r_{1}^{2}=r_{2}^{2}=10^{-4}$ and $l=1.5 \mathrm{~mm}$.

nonzero $\alpha$-parameter. The phase noise spectra in Fig. 9 overall show increased broadening as the chip gain is increased in correspondence with the RIN.

As in [11], [23] we have omitted the dc part of the output when calculating the field power spectrum for an SOA. When calculating field power spectra for an injection-locked laser in Section VIII, we shall include an input with a finite spectral linewidth and preserve the dc part of the spectra. Fig. 10 displays the field power spectra corresponding to Figs. 8 and 9. The field power spectra for the lowest gain case of $14.2 \mathrm{~dB}$ has an asymmetric shape in agreement with [11], while broadened and more complicated structure is seen for increasing values of chip gain, partly as a consequence of the reflections at the facets.

\section{INJECTION-LOCKED LASER}

When the end-facet reflectivities become sufficiently high, an active waveguide subject to injection of light can act as an injection-locked laser. Here, we show examples of responses and field power spectra for a laser with light injection. When injecting light into a semiconductor laser the cavity modes of the solitary laser will be shifted because of a change in carrier density and the frequency of the shifted cavity mode need not be identical to the frequency of the injected signal for locking to 


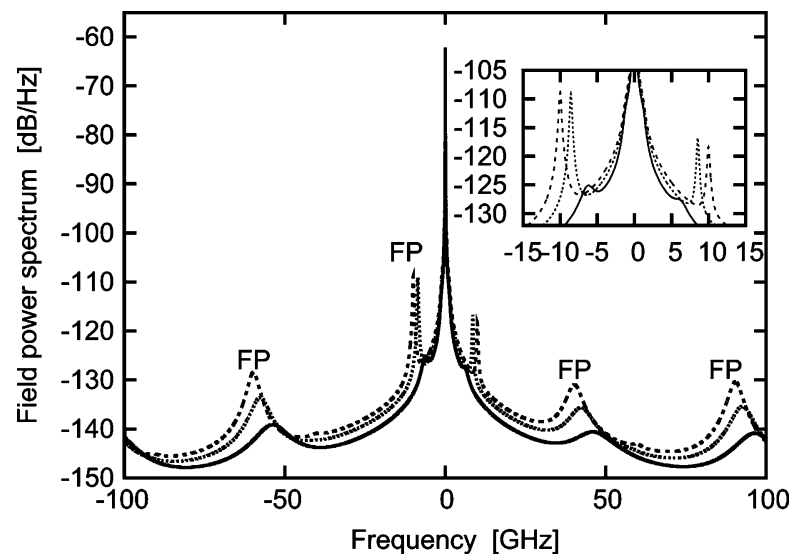

Fig. 11. Normalized field power spectrum, $S_{E, E}(\Omega) /\left|u_{s}^{+}(l)\right|^{2}$. The curves are for detunings $\Delta=35 \mathrm{GHz}$ (solid), $\Delta=9 \mathrm{GHz}$ (dashed), and $\Delta=1 \mathrm{GHz}$ (dotted). Slave laser has reflectivities $r_{1}^{2}=r_{2}^{2}=10^{-2}$ and length $l=750 \mu \mathrm{m}$. "FP" denotes groups of each three cavity modes. Inset shows close-up: structure visible for positive frequencies are due to four-wave mixing.

occur. It has been demonstrated that a resonance in the modulation response can be achieved at a baseband frequency equal to the difference in frequency between the injected signal and the nearest shifted cavity mode, when the cavity mode is on the red side of the injected signal [4], [15]. Equivalently, the carrier dynamics can be modified by the injected light potentially giving a modulation-resonance frequency higher than in the absence of injected light [26]. Moreover, injection of an RF-intensity modulated optical signal with an optical carrier detuned from the cavity frequency into a VCSEL has been seen to give rise to RF-phase shifts of 360 degrees [16].

Since the spatial extent of the cavity is included in the calculations presented here, the presence of multiple longitudinal modes can be seen in spectra and response curves. For three different values of the detuning $\Delta$ [see (2)] leading to three different detunings between the injected signal and the nearest shifted cavity mode, we show the field power spectrum in Fig. 11 as calculated from (52). Here the injected signal has the baseband frequency $\Omega / 2 \pi=0$. The carrier signal has an input power of $P_{\mathrm{in}}^{+}=0.1 \mathrm{~mW}$. The linewidth of the incident signal from the master laser is taken to be finite, namely $60 \mathrm{MHz}$. The noise spectra of the master laser is assumed to be the spectra of a single mode semiconductor laser with its frequency noise spectrum [27] being dominant. In Fig. 11 the full line denotes the case of $\Delta=35 \mathrm{GHz}$ where the nearest shifted cavity mode lies $6.2 \mathrm{GHz}$ below the master frequency, the dashed curve represents $\Delta=9 \mathrm{GHz}$ for which the nearest cavity mode is $10.0 \mathrm{GHz}$, and finally for $\Delta=1 \mathrm{GHz}$ the nearest shifted cavity mode is at $8.5 \mathrm{GHz}$ below the injected signal frequency (dotted line). Three other cavity modes are present in the shown parts of the spectra for each value of $\Delta$. In Fig. 11, the cavity modes lie in groups of three each marked "FP" for Fabry-Perot. The length of the slave laser is here $l=750 \mu \mathrm{m}$ corresponding to a mode spacing of $50 \mathrm{GHz}$. Other small spikes are four-wave mixing images of the cavity modes. Note that since no gain dispersion is considered, the system is periodic as a function of $\Delta$, where the frequency of periodicity is given

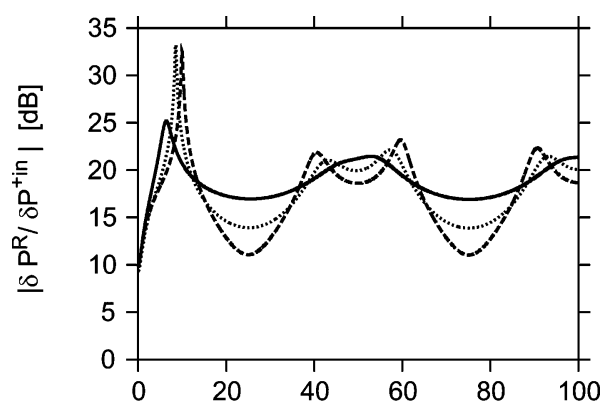

(a)

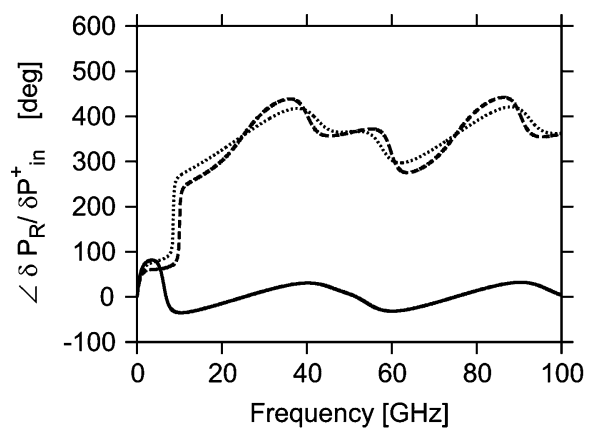

(b)

Fig. 12. Modulation responses for cases in Fig. 11. (a), (b) Magnitude and argument of the power response due to optical power modulation. The curves are for $\Delta=35 \mathrm{GHz}$ (solid), $\Delta=9 \mathrm{GHz}$ (dashed), and $\Delta=1 \mathrm{GHz}$ (dotted)

by the mode spacing. Here, $\Delta=35 \mathrm{GHz}$ is thus equivalent to $\Delta=-15$ GHz. It should also be noted that in this paper we do not state the condition for phase-locking. Instead, we leave such an analysis of the locking bandwidth for future work.

For a single-mode laser, the cavity mode of the freely running laser is shifted towards lower frequencies when light is injected into the laser. It has been shown that, when the difference in frequency between the injected signal and the cavity mode is positive, then under current modulation of the injected-locked signal there can be a resonance at a modulation frequency corresponding to this frequency difference [15], [26]. It has been argued that, of the two symmetric sidebands generated due to a modulation, the one on the red side of the injected signal is more strongly amplified when located at the frequency of the cavity mode [28]. Examples of modulation responses for the three different detunings in Fig. 11 are shown in Figs. 12-14. First, in Figs. 12-13 responses due to modulation of the optical input signal are given. The magnitude $\left|\delta P_{R} / \delta P_{\text {in }}^{+}\right|$displays resonances at $6.2,8.5$, and $10.0 \mathrm{GHz}$ for the respective three detunings in correspondence with the spectrum in Fig. 11(a), where the cavity mode nearest the injected signal has negative baseband frequencies. This resonance is related to the interaction between the charge carriers and photon population. The next resonance appears around $40 \mathrm{GHz}$ (for $\Delta=1 \mathrm{GHz}$ and $\Delta=9 \mathrm{GHz}$ ). This is a higher order resonance whose location is given by the beat frequency of the difference between the master frequency and the nearest cavity mode (here for the two clearest cases $8.5 \mathrm{GHz}$ and $10.0 \mathrm{GHz}$ ) and the mode-spacing (here $50 \mathrm{GHz}$ ). The next higher frequency resonances are given by the sum of the detuning and the cavity mode spacing (here for the two clear cases have peaks around $60 \mathrm{GHz}$ ). For $\Delta=$ 


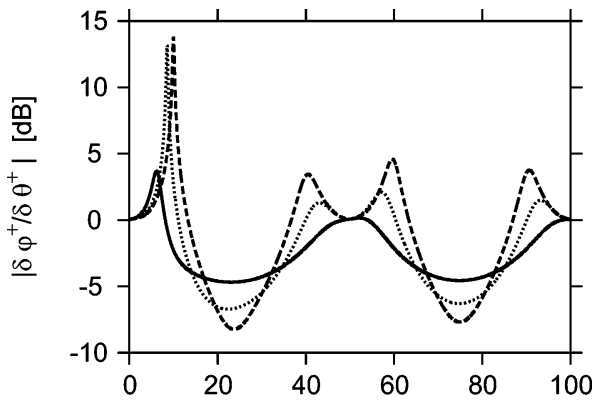

(a)

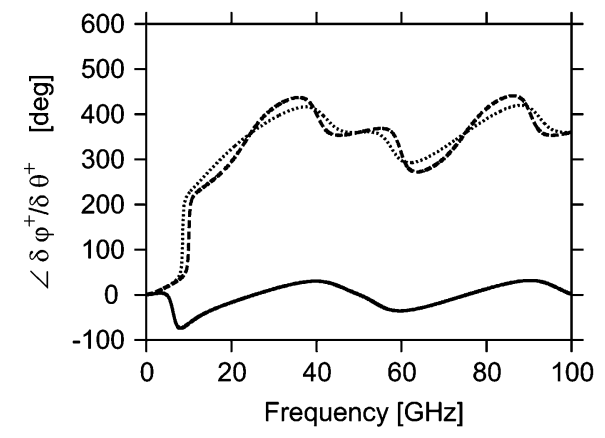

(b)

Fig. 13. Modulation responses for cases in Fig. 11. (a) and (b) Magnitude and argument of the optical phase response due to optical phase modulation. The curves are for $\Delta=35 \mathrm{GHz}$ (solid), $\Delta=9 \mathrm{GHz}$ (dashed), and $\Delta=1 \mathrm{GHz}$ (dotted).

$35 \mathrm{GHz}$ the beat frequency is $43.8 \mathrm{GHz}$ and the two soft resonances (here the other one at 56.2) merge into one broad peak with maximum around $53 \mathrm{GHz}$. Finally, the resonances around $90 \mathrm{GHz}$ can be understood as beat frequencies involving two times the mode spacing (i.e., $100 \mathrm{GHz}$ ). The higher order resonances are thus related to the round-trip time of the photons in the cavity. Similar higher order modulation resonances can be found in long cavity DBR lasers without optical injection [29], [30].

The behavior of the response $\left|\delta \varphi^{+} / \delta \theta^{+}\right|$in Fig. 13(a) is qualitatively very similar to Fig. 12(a). Modulating the optical phase $\theta^{+}$thus gives rise to a response in optical phase at the output, which can be greater than $0 \mathrm{~dB}$. In Fig. 14(a), the magnitude of the power response following a current modulation $\left|\delta P_{R} / \delta I_{z}\right|$ has resonances at the same modulation frequencies as seen for the optical modulation formats.

The corresponding arguments of the responses are displayed in Figs. 12(b)-14(b). By changing the detuning $\Delta$ it possible to obtain a RF-phase change of 360 degrees for modulation frequencies above the lowest resonance frequencies. The behavior of the response $\angle \delta P_{R} / \delta P_{\text {in }}^{+}$shown in Fig. 12(b) has been observed in a VCSEL [8]. In Fig. 13(b), we see a very similar similar appearance of the response $\angle \delta \varphi^{+} / \delta \theta^{+}$. The main qualitative difference is apparent at low frequencies where in Fig. 12(b) a significant positive argument due to carrier fluctuations is seen as opposed to Fig. 13(b) where the argument is close to zero degrees. Finally, in Fig. 14(b), even in the case of a current modulation uniformly distributed over $z=0$ to $z=l$, we see the possibility of a change in argument of 360 degrees when varying $\Delta$.

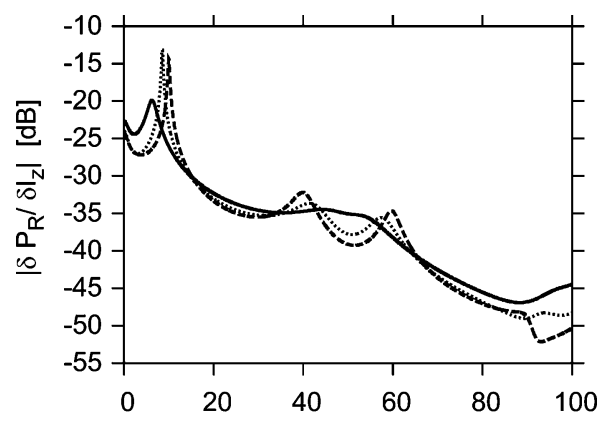

(a)

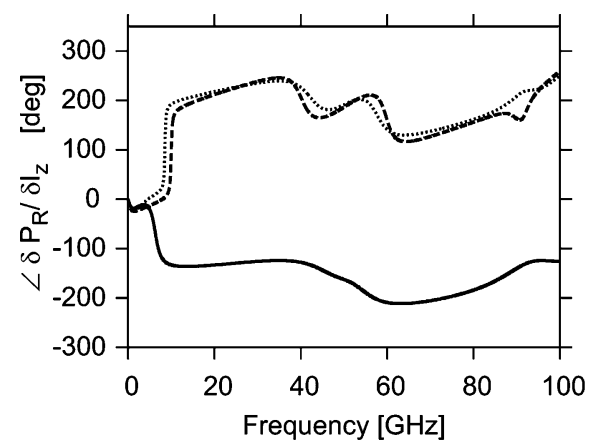

(b)

Fig. 14. Modulation responses for cases in Fig. 11. (a), (b) Magnitude and argument of the power response due to a uniform current modulation. The curves are for $\Delta=35 \mathrm{GHz}$ (solid), $\Delta=9 \mathrm{GHz}$ (dashed), and $\Delta=1 \mathrm{GHz}$ (dotted).

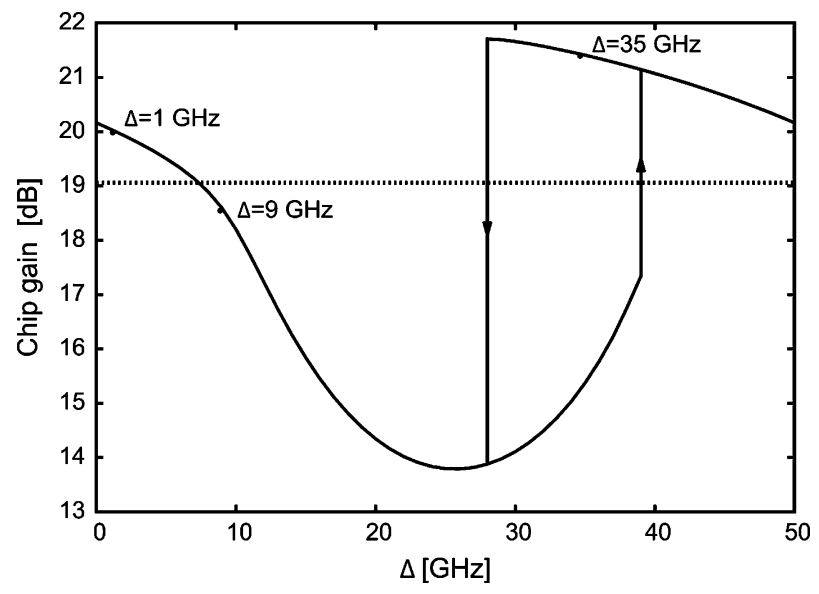

Fig. 15. Calculated stable stationary solutions for a laser with light injection depicted as curves in the chip gain versus detuning plane. The three bullets correspond to the stationary solutions used for computing responses in Figs. 12-14. The horizontal dotted line indicates the threshold gain of the solitary slave laser.

While the modulation responses in Figs. 12-14 show pronounced resonance peaks whose frequencies are given by the relaxation oscillation frequency and its beating with multiples of the cavity resonance frequency, the strongest features of the responses for an SOA shown in Figs. 2-5 are chiefly a product of the carrier lifetime albeit modified by the reflections at the mirrors.

The three stationary solutions with $\Delta$-values of 1,9 , and $35 \mathrm{GHz}$ are represented by the bullets in the chip gain versus $\Delta$ curve in Fig. 15. The laser exhibits bistability as the detuning $\Delta$ is varied for the fixed injection level. Bistable behavior of 
semiconductor lasers with light injection can be found both experimentally and theoretically [31], [32]. Here we find that stationary solutions with chip gain relatively close to the threshold gain of the solitary slave laser may yield large positive RF-phase shifts, here exemplified by $\Delta=1 \mathrm{GHz}$ and $\Delta=9 \mathrm{GHz}$. Stationary solutions with chip gain relatively far from the threshold gain, here exemplified by $\Delta=35 \mathrm{GHz}$, tend to have small positive or negative RF-phase shifts.

\section{CONCLUSION}

We have presented a method for calculating small-signal modulation responses and noise spectra of an active Fabry-Perot semiconductor waveguide subject to external injection of light. The method includes the longitudinal variation of carrier density in the device and reflections from end facets. By using a Green's function technique, semi-analytical small-signal expressions were derived for responses and spectra. As examples of the versatility of the method, responses and noise spectra were calculated for SOAs. For instance, the modulation-frequency dependence of the RF-phase shift in an SOA (slow/fast light) was susceptible to modifications due to reflections at the facets. It was also demonstrated how a phase modulated optical input signal can cause nontrivial responses in an active waveguide when the end-facet reflectivities are finite. Further, the method was used to calculate spectra and responses of a laser with light injection, something that is usually done using a lumped model of the slave laser. Inspection of the calculated field power spectra led to an understanding of the resonances seen in the responses. When varying the detuning between the master and the slave laser, large RF-phase shifts of 360 degrees, previously seen in VCSELS with light injection, was found for a much longer edge-emitting device.

\section{APPENDIX A \\ GAIN MODEL}

For the example calculations presented in this paper, a model describing gain in quantum wells has been utilized. The usual expression for material gain for a two-level system (see [27, eq. (4.37)]) is supplemented by a secant lineshape function $L(\Delta E)=\eta \tau / 2 \hbar \operatorname{sech}^{2}(\eta \tau / \hbar \Delta E)$ where $\Delta E$ is the deviation from the transition energy, $\eta$ is the relative half width $\eta=\log (3+2 \sqrt{2})$, and $\tau$ is the dephasing time (here taken as $50 \mathrm{fs}$ ). The two-level gain function is convolved with the lineshape function to obtain the material gain including lineshape broadening (see [27, eq. (4.40)]). The considered 2-QW structure consists of wells of thickness $7 \mathrm{~nm}$, with effective hole mass $0.37 m_{0}$ and effective electron mass $0.045 m_{0}$, and a transition matrix element of $7.83 \cdot 10^{-49} \mathrm{~J} \cdot \mathrm{kg}$ [27]. Only two bands are considered. The Fermi energies are calculated for the lowest order discrete energy level of the valence band and conduction band, respectively.

Nonlinear gain is introduced in the relation between the modal gain $g$ and the material gain $g_{m}$ where the confinement factor $\Gamma$ and the nonlinear gain coefficient $\epsilon$ are used as follows:

$$
g(\omega, N, S)=\frac{\Gamma g_{m}(\omega, N)}{1+\epsilon S}
$$

The power due to ASE entering the carrier equation is said to be divided into a number of frequency intervals of bandwidth $\Delta f[20]$. Each interval around frequency $f_{j}$ is governed by equations for the forward and backward traveling ASE governed by the equations (one for each direction)

$$
\begin{aligned}
\frac{\partial}{\partial z} P_{\mathrm{sp}}^{ \pm}\left(f_{j}\right)= \pm\left(\Gamma g_{m}-\right. & \left.\alpha_{i}\right) P_{\mathrm{sp}}^{ \pm}\left(f_{j}\right) \\
& +\Gamma h f_{j} g_{m}(\omega, N) n_{\mathrm{sp}}\left(f_{j}, N\right) \Delta f
\end{aligned}
$$

where $n_{\mathrm{sp}}$ is the population inversion factor and $\Delta f$ is the optical bandwidth. At the end facets, the boundary conditions for $P_{\mathrm{ASE}}^{ \pm}$are expressed as

$$
\begin{aligned}
P_{\mathrm{ASE}}^{+}(0) & =\left|r_{1}\right|^{2} P_{A S E}^{-}(0)+\left(1-\left|r_{1}\right|^{2}\right) P_{\mathrm{ASE}, \mathrm{in}}^{+} \\
P_{\mathrm{ASE}}^{-}(l) & =\left|r_{2}\right|^{2} P_{\mathrm{ASE}}^{+}(l)+\left(1-\left|r_{2}\right|^{2}\right) P_{\mathrm{ASE}, \text { in }}^{-}
\end{aligned}
$$

The recombination rate in connection with stimulated emission due to ASE is written

$$
R_{\mathrm{ASE}}=v_{g} \sum_{i=1,2} \sum_{j} K_{i} g_{m}\left(N, f_{j}\right) S_{\mathrm{ASE}}\left(f_{j}\right) .
$$

The photon density $S_{\mathrm{ASE}}$ is given as $S_{\mathrm{ASE}}=\left(P_{\mathrm{ASE}}^{+}+\right.$ $\left.P_{\mathrm{ASE}}^{-}\right) /\left(v_{g} \hbar \omega A_{\mathrm{ph}}\right)$. The constants $K_{i}$ with $i=1$ for TE and $i=2$ for TM polarization are weights for the two different polarizations of light. For bulk material they are unity for both polarizations, while for QWs the gain is strongly polarized giving different weights. For QWs it is fair to make the approximation $K_{1}=1$ and $K_{2}=0$.

\section{APPENDIX B MATRICES}

The Laplace transform of the differential $\delta N(t, z)$ in (10) may be written as

$$
\begin{aligned}
\widetilde{\delta N}(s, z)=\frac{\tau_{R}}{1+s \tau_{R}}[\widetilde{\delta J}(s, z) & +F_{N} \\
& \left.-\left(\frac{\partial R_{\text {st }}}{\partial S}+\frac{\partial R_{\text {ase }}}{\partial S}\right) \boldsymbol{b}^{T} \boldsymbol{x}\right]
\end{aligned}
$$

where the vector $\boldsymbol{b}$ is defined as $\boldsymbol{b}=2\left(S_{s}^{+}, 0, S_{s}^{-}, 0\right)^{T}$. The matrix $\boldsymbol{M}_{0}$ in (10) follows as

$$
\boldsymbol{M}_{0}=\left[\left(\frac{\partial R_{\mathrm{st}}}{\partial S}+\frac{\partial R_{\mathrm{ase}}}{\partial S}\right) \boldsymbol{h}_{N}-\boldsymbol{h}_{S}\right] \boldsymbol{b}^{T}+s \boldsymbol{M}_{\omega}
$$

where

$$
\boldsymbol{h}_{N}=\frac{v_{g}}{2} \frac{\partial g}{\partial N}(z) \frac{\tau_{R}}{1+s \tau_{R}}(1, \alpha, 1, \alpha)^{T}
$$

and

$$
\boldsymbol{h}_{S}=\frac{v_{g}}{2} \frac{\partial g}{\partial S}(z)\left(1, \alpha_{S}, 1, \alpha_{S}\right)^{T} .
$$

In (62), $\alpha=-\operatorname{Re}(\partial k / \partial N) / \operatorname{Im}(\partial k / \partial N)$ is the linewidth enhancement factor. It has been assumed that $\alpha_{s}=$ $-\operatorname{Re}(\partial k / \partial S) / \operatorname{Im}(\partial k / \partial S)=0$. When the signal experiences no gain dispersion, the matrix $\boldsymbol{M}_{\omega}$ is simply the unit matrix. Further, the matrix $\boldsymbol{M}_{1}$ is diagonal matrix with $\left(\boldsymbol{M}_{1}\right)_{11}=\left(\boldsymbol{M}_{1}\right)_{22}=-\left(\boldsymbol{M}_{1}\right)_{33}=-\left(\boldsymbol{M}_{1}\right)_{44}=v_{g}$. The 
partial derivatives $\partial g / \partial N$ and $\partial g / \partial S$ in general depend on $z$. Finally, the Langevin driving vector function in (18) becomes

$$
\widetilde{\boldsymbol{f}}=\left(\widetilde{F}_{r}^{+}, \widetilde{F}_{i}^{+}, \widetilde{F}_{r}^{-}, \widetilde{F}_{i}^{-}\right)^{T}+\boldsymbol{h}_{N} \widetilde{F}_{N}
$$

where $F_{r}$ and $F_{i}$ are given by

$$
\begin{aligned}
& F_{r}^{ \pm}=\frac{v_{g}}{2}\left\{\frac{f^{ \pm}}{u_{s}^{ \pm}}+\text {c.c. }\right\} \\
& F_{i}^{ \pm}=\frac{v_{g}}{2 j}\left\{\frac{f^{ \pm}}{u_{s}^{ \pm}}-\text {c.c. }\right\} .
\end{aligned}
$$

The diffusion coefficient for carrier density fluctuations $D_{N N}$ in the correlation relation for $F_{N}$

$$
\begin{aligned}
&\left.\left\langle F_{N}(s, z), F_{N}\left(s^{\prime}, z^{\prime}\right)\right\rangle\right) \\
&=2 D_{N N}(z) \delta\left(z-z^{\prime}\right) 2 \pi \delta\left(\Omega-\Omega^{\prime}\right)
\end{aligned}
$$

is given by

$$
2 D_{N N}=\frac{1}{A_{c}}\left\{\xi J+R+\left(2 n_{\mathrm{sp}}-1\right) R_{\mathrm{st}}\right\} .
$$

Here, $\xi$ describes the noise of the current pump source. For $\xi=$ 1 the pump source exhibits shot noise behavior, while for $\xi<1$ it behaves sub-Poissonian. In this paper, $\xi=1$ is used.

\section{APPENDIX C \\ GREEN's FUNCTION $\zeta_{i}\left(s, z^{\prime}, z\right)$}

The expression (18) is obtained using partial integration and by choosing the appropriate conditions for the vector Green's function $\zeta_{i}\left(s, z^{\prime}, z\right)$ as shown in this appendix. Partial integration gives

$$
\begin{aligned}
\int_{0}^{l} & \boldsymbol{\zeta}_{i}^{\dagger}\left(s, z^{\prime}, z\right)\left[\boldsymbol{M}_{0}\left(s, z^{\prime}\right)+\boldsymbol{M}_{1}\left(z^{\prime}\right) \frac{\partial}{\partial z^{\prime}}\right] \boldsymbol{x}\left(s, z^{\prime}\right) d z^{\prime} \\
= & \int_{0}^{l}\left\{\left[\boldsymbol{M}_{0}^{\dagger}\left(s, z^{\prime}\right)-\frac{\partial}{\partial z^{\prime}} \boldsymbol{M}_{1}\left(z^{\prime}\right) \frac{\partial}{\partial z^{\prime}}\right] \boldsymbol{\zeta}_{i}\left(s, z^{\prime}, z\right)\right\}^{\dagger} \\
& \times \boldsymbol{x}\left(s, z^{\prime}\right) d z^{\prime}+\left[\left(\boldsymbol{M}_{1}^{\dagger}\left(z^{\prime}\right) \boldsymbol{\zeta}_{i}\left(s, z^{\prime}, z\right)\right)^{\dagger} \boldsymbol{x}\left(s, z^{\prime}\right)\right]_{0}^{l} \\
= & \int_{0}^{l} \delta\left(z^{\prime}-z\right) \boldsymbol{e}_{i}^{T} \boldsymbol{x}\left(s, z^{\prime}\right) d z^{\prime} \\
& +\left[\left(\boldsymbol{M}_{1}^{\dagger}\left(z^{\prime}\right) \boldsymbol{\zeta}_{i}\left(s, z^{\prime}, z\right)\right)^{\dagger} \boldsymbol{x}\left(s, z^{\prime}\right)\right]_{0}^{l} \\
= & \boldsymbol{x}(s, z)+\left[\left(\boldsymbol{M}_{1}^{\dagger}\left(z^{\prime}\right) \boldsymbol{\zeta}_{i}\left(s, z^{\prime}, z\right)\right)^{\dagger} \boldsymbol{x}\left(s, z^{\prime}\right)\right]_{0}^{l}
\end{aligned}
$$

Boundary conditions for $\boldsymbol{x}$ in (19) and $\boldsymbol{\zeta}_{i}$ in (21) at $z=0$ and $z=l$ give the relations

$$
\begin{aligned}
& {\left[\boldsymbol{M}_{1}^{\dagger}(0) \zeta_{i}(s, 0, z)\right]^{\dagger} \boldsymbol{x}(s, 0)} \\
& \quad=\left(\left[\boldsymbol{M}_{1}^{\dagger}(0) \zeta_{i}(s, 0, z)\right]^{\dagger}\right)_{1}\left(\widetilde{\boldsymbol{v}}_{0}\right)_{1} \\
& \quad+\left(\left[\boldsymbol{M}_{1}^{\dagger}(0) \boldsymbol{\zeta}_{i}(s, 0, z)\right]^{\dagger}\right)_{2}\left(\widetilde{\boldsymbol{v}}_{0}\right)_{2}
\end{aligned}
$$

$$
\begin{aligned}
& {\left[\boldsymbol{M}_{1}^{\dagger}(l) \boldsymbol{\zeta}_{i}(s, l, z)\right]^{\dagger} \boldsymbol{x}(s, l)} \\
& =\left(\left[\boldsymbol{M}_{1}^{\dagger}(l) \boldsymbol{\zeta}_{i}(s, l, z)\right]^{\dagger}\right)_{3}\left(\widetilde{\boldsymbol{v}}_{0}\right)_{3} \\
& \quad+\left(\left[\boldsymbol{M}_{1}^{\dagger}(l) \boldsymbol{\zeta}_{i}(s, l, z)\right]^{\dagger}\right)_{4}\left(\widetilde{\boldsymbol{v}}_{0}\right)_{4} .
\end{aligned}
$$

Then, by multiplying both sides of (18) by $\zeta_{i}^{\dagger}\left(s, z^{\prime}, z\right)$ and integrating from $z=0$ to $z=l$, utilizing (69) and (70) leads to the explicit expression for $\boldsymbol{x}$ in (22).

\section{ACKNOWLEDGMENT}

The authors wish to thank B. Tromborg, F.Öhman, and I. T. Monroy, DTU Fotonik, for fruitful discussions.

\section{REFERENCES}

[1] S. Kobayashi and T. Kimura, "Optical phase modulation in an injection locked AlGaAs semiconductor laser," IEEE J. Quantum Electron., vol. QE-18, no. 10, pp. 1662-1669, Oct. 1982.

[2] K. Kikuchi, T. Okoshi, and S. Tanikoshi, "Amplitude modulation of an injection-locked semiconductor laser for heterodyne-type optical communications," Opt. Lett., vol. 9, no. 3, pp. 99-101, 1984.

[3] T. Simpson, J. Liu, and A. Gavrielides, "Enhanced modulation bandwidth in injection-locked semiconductor lasers," IEEE J. Quantum Electron., vol. 32, no. 8, pp. 1456-1468, Aug. 1996.

[4] A. Murakami, K. Kawashima, and K. Atsuki, "Cavity resonance shift and bandwidth enhancement in semiconductor lasers with strong light injection," IEEE J. Quantum Electron., vol. 39, no. 10, pp. 1196-1204, Oct. 2003.

[5] J. Mørk, A. Meccozi, and G. Eisenstein, "The modulation response of a semiconductor laser amplifier," IEEE J. Sel. Topics Quantum Electron., vol. 5, no. 3, pp. 851-860, May/Jun. 1999.

[6] T. N. Nielsen, N. Storkfelt, U. Gliese, B. Mikkelsen, T. Durhuus, K. E. Stubkjaer, B. Fernier, F. Leblond, and A. Accard, "Cancelation of inherent AM in semiconductor optic phase modulators," Electron. Lett., vol. 28, no. 3, pp. 235-236, 1992.

[7] A. Uskov and C. Chang-Hasnain, "Slow and superluminal light in semiconductor optical amplifiers," Electron. Lett., vol. 41, no. 16, pp. $55-56,2005$.

[8] H. Su, P. Kondratko, and S. L. Chuang, "Variable optical delay using population oscillation and four-wave-mixing in semiconductor optical amplifiers," Opt. Exp., vol. 14, no. 11, pp. 4800-4807, 2006.

[9] J. Mørk, R. Kjaer, M. van der Poel, and K. Yvind, "Slow light in a semiconductor waveguide at Gigahertz frequencies," Opt. Exp., vol. 13, no. 20, pp. 8136-8145, 2005.

[10] F. Ohman, B. Tromborg, and J. Mørk, "Output power PDF of a saturated semiconductor optical amplifier: Second-order noise contributions by path integral method," IEEE J. Quantum Electron., vol. 43, no. 12, pp. 1188-1197, Dec. 2007.

[11] M. Shtaif, B. Tromborg, and G. Eisenstein, "Noise spectra of semiconductor optical amplifiers: Relation between semiclassical and quantum descriptions," IEEE J. Quantum Electron., vol. 34, no. 5, pp. 869-878, May 1998.

[12] R. Lang, "Injection locking properties of a semiconductor laser," IEEE J. Quantum Electron., vol. QE-18, no. 6, pp. 976-983, Jun. 1982.

[13] F. Mogensen, H. Olesen, and G. Jacobsen, "Locking conditions and stability properties for a semiconductor laser with external light injection," IEEE J. Quantum Electron., vol. QE-21, no. 7, pp. 784-793, Jul. 1985.

[14] S. Piazolla, P. Spano, and M. Tamburrini, "Small signal anaylysis of frequency chirping in injection-locked semiconductor lasers," IEEE J. Quantum Electron., vol. QE-22, no. 12, pp. 2219-2223, Dec. 1986.

[15] H.-K. Sung, E. K. Lau, and M. C. Wu, "Optical properties and modulation characteristics of ultra-strong injection-locked distributed feedback lasers," IEEE J. Sel. Topics Quantum Electron., vol. 13, no. 5, pt. 1, pp. 1215-1221, Sep./Oct. 2007.

[16] X. Zhao, Y. Zhou, C. J. Change-Hasnain, W. Hofmann, and M. C. Amann, "Novel modulated-master injection-locked $1.55-\mu \mathrm{m}$ VCSELs," Opt. Exp., vol. 14, no. 22, pp. 10500-10507, 2006.

[17] Y.-Y. Won, H.-C. Kwon, S.-K. Han, E.-S. Jung, and B.-W. Kim, "OBI noise reduction using gain saturated SOA in reflective SOA based WDN/SCM-PON optical links," Electron. Lett., vol. 42, no. 17, pp. 992-993, 2006. 
[18] Y. Awaji, T. Kuri, and W. Chujo, "Direct detection of OC-192 DPSK signal using polarization-controlled differential-phase-to-intensity conversion (DPIC)," IEEE Photon. Technol. Lett., vol. 14, no. 7, pp. 1007-1009, Jul. 2002.

[19] B. Tromborg, H. E. Lassen, and H. Olesen, "Traveling wave analysis of semiconductor lasers: Modulation responses, mode stability and quantum mechanical treatment of noise spectra," IEEE J. Quantum Electron., vol. 30, no. 4, pp. 939-956, Apr. 1994.

[20] T. Durhuus, B. Mikkelsen, and K. E. Stubkjaer, "Detailed dynamic model for semiconductor optical amplifiers and their crosstalk and intermodulation distortion," IEEE J. Quantum Electron., vol. 28, no. 4, pp. 1056-1065, Apr. 1992.

[21] K. Yvind, "Semiconductor mode-locked lasers for optical communication systems," Ph.D. dissertation, Research Center COM, Technical Univ. of Denmark, Lyngby, 2003.

[22] L. J. Christiansen, "Optical semiconductor components," Ph.D. dissertation, Technical Univ. of Denmark, Research Center COM, , Lyngby, 2006.

[23] M. Shtaif and G. Eisenstein, "Noise characteristics of nonlinear semiconductor optical amplifiers in the Gaussian limit," IEEE J. Quantum Electron., vol. 32, no. 10, pp. 1801-1809, Oct. 1996.

[24] S. Blaaberg, P. M. Petersen, and B. Tromborg, "Structure, stability, and spectra of lateral modes of a broad-area laser," IEEE J. Quantum Electron., vol. 43, no. 11, pp. 959-973, Nov. 2007.

[25] E. Detoma, B. Tromborg, and I. Montrosset, "The complex way to laser diode spectra: Example of an external cavity laser with strong optical feedback," IEEE J. Quantum Electron., vol. 41, no. 2, pp. 171-181, Feb. 2005.

[26] T. Simpson and J. Liu, "Enhanced modulation bandwidth in injectionlocked semiconductor lasers," IEEE Photon. Technol. Lett., vol. 9, no. 9, pp. 1322-1324, Sep. 1997.

[27] L. A. Coldren and S. W. Corzine, Diode Lasers and Photonic Integrated Circuits. New York: Wiley, 1995.

[28] H.-K. Sung, E. K. Lau, and M. C. Wu, "Optical single sideband modulation using strong optical injection-locked semiconductor lasers," IEEE Photon. Technol. Lett., vol. 19, no. 13, pp. 1005-1007, Jul. 2007.
[29] L. Bach, W. Kaiser, J. Reithmaier, A. Forchel, M. Gioannini, V. Feies, and I. Montrosset, " 22 -GHz modulation bandwidth of long cavity DBR laser by using a weakly laterally coupled grating fabricated by focused ion beam lithography," IEEE Photon. Technol. Lett., vol. 16, no. 1, pp. 18-20, Jan. 2004

[30] G. Morthier, R. Schatz, and O. Kjebon, "Extended modulation bandwidth of DBR and external cavity lasers by utilizing a cavity resonance for equalization," IEEE J. Quantum Electron., vol. 36, no. 12, pp. $1468-1475$, Dec. 2000

[31] H. Kawaguchi, K. Inoue, T. Matsuaka, and K. Otsuka, "Bistable output characteristics in semiconductor laser injection locking," IEEE J. Quantum Electron., vol. QE-21, no. 8, pp. 1314-1317, Aug. 1985.

[32] J. Mørk and B. Tromborg, "Nonlinear injection locking dynamics and the onset of coherence collapse in the external cavity lasers," IEEE J. Quantum Electron., vol. 26, no. 4, pp. 642-654, Apr. 1990.

Søren Blaaberg received the M.Sc. degree in engineering physics and the Ph.D. degree from the Technical University of Denmark (DTU), Lyngby, in 2002 and 2007, respectively.

He is now a Postdoctoral Research Fellow with DTU Fotonik at the Technical University of Denmark. His current research interests include the physics of optical semiconductor devices and nonlinear dynamics in spatially extended systems.

Jesper Mørk received the Ph.D. and Dr. Techn. degrees from the Technical University of Denmark (DTU), Lyngby, in 1988 and 2003, respectively.

Since 2002, he has been a Professor with DTU Fotonik, DTU. His current research interests are in the area of device physics, in particular, ultrafast devices for optical signal processing, noise in nonlinear devices, and quantum photonics. 